\title{
S. cerevisiae synthetic transcriptional networks harnessing dCas12a and type V-A anti-CRISPR proteins.
}

\section{Supplementary Data}

\author{
Lifang $\mathrm{Yu}^{1}$ and Mario Andrea Marchisio ${ }^{1, *}$ \\ ${ }^{1}$ School of Pharmaceutical Science and Technology, Tianjin University, 300072 Tianjin, China \\ * To whom correspondence should be addressed. Email: mario@tju.edu.cn, mamarchisio@yahoo.com.
}

\section{Supplementary Figures}

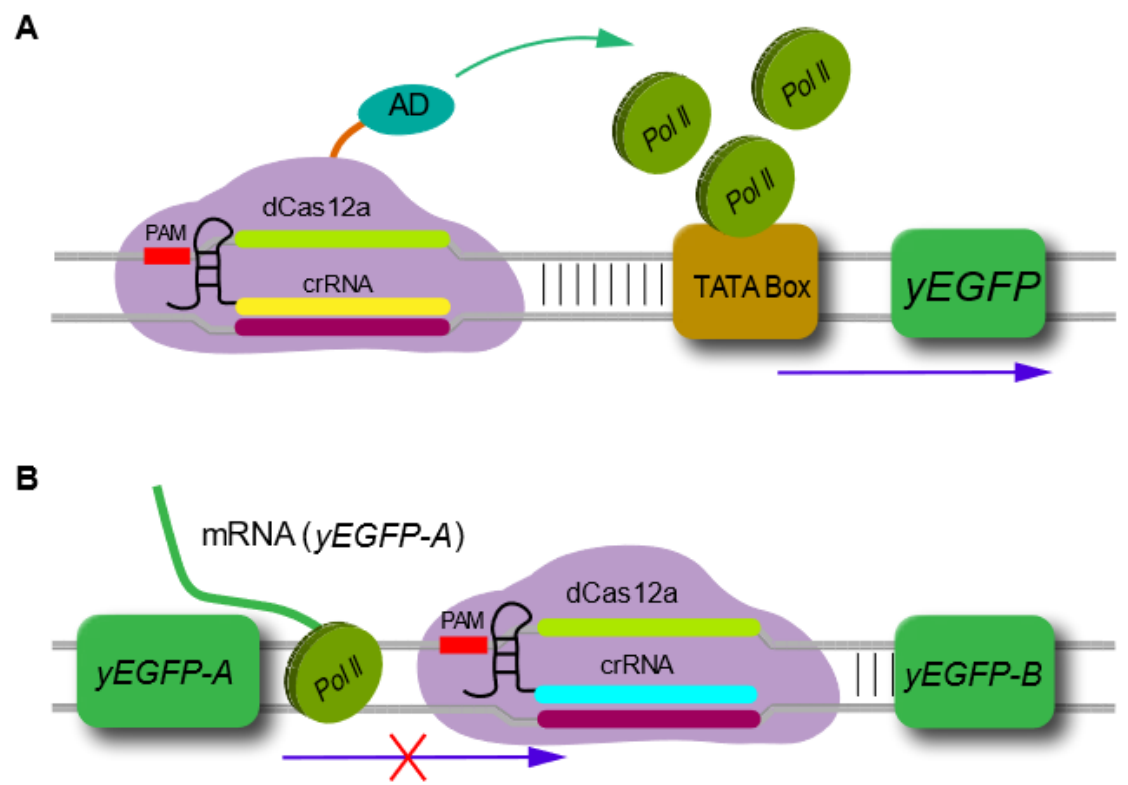

Figure S1. Transcriptional controls based on of dCas12a. (A) Activation. An activation domain $(A D)$ is fused to dCas12a in order to recruit RNA polymerase II (Pol II) to the DNA. The complex dCas12a-AD:crRNA binds a sequence-complementary to the spacer of the crRNA-just downstream of the protospacer adjacent motif (PAM) and several nucleotides upstream of the promoter core sequence, where the TATA box lies-the anchor point of Pol II. In this configuration, the presence of dCas12a-AD:crRNA on the DNA increases the flux of Pol II that binds the TATA box and starts mRNA synthesis. Here, we suppose that the yeast enhanced green fluorescent protein gene ( $y E G F P)$ is transcribed. (B) Repression. The complex dCas12a:crRNA binds, around the middle of $y E G F P$, a novel DNA target placed downstream of the usual TTTV PAM. Pol II molecules, which already transcribed the $y E G F P-A$ gene section, clash against dCas12a and fall from the DNA releasing, in this way, a truncated transcript. 


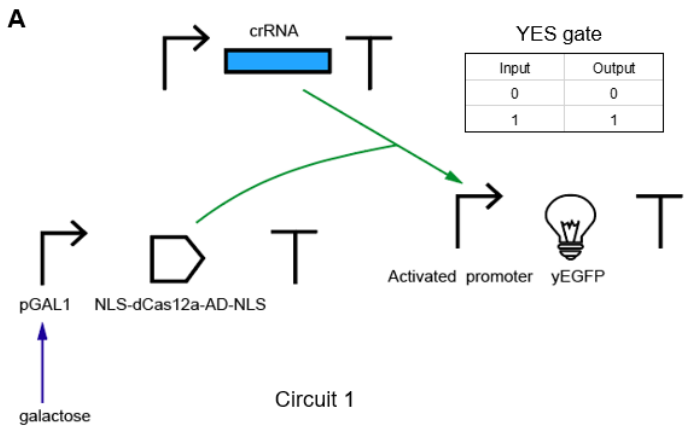

C

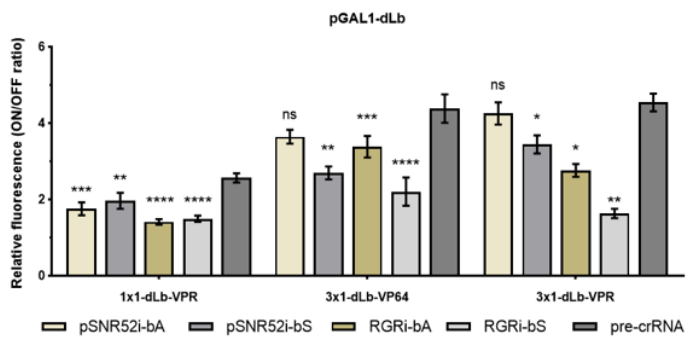

B

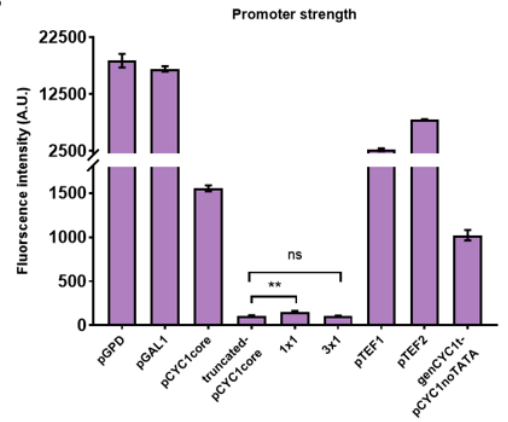

D

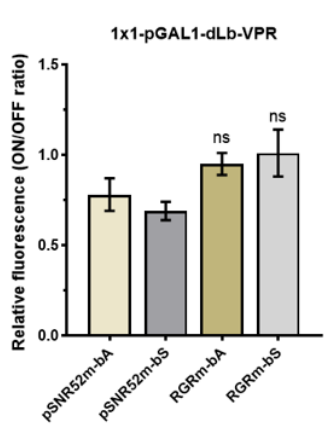

E

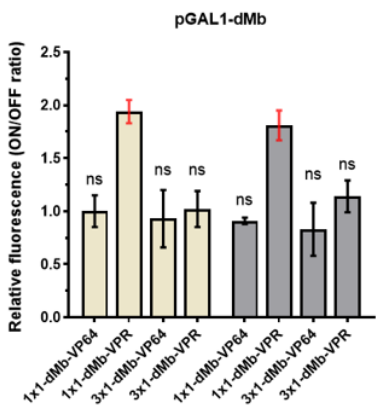

Figure S2. YES gates. A) Circuit 1 diagram, as reported in the main text. B) Strength of the promoters used in our work. The two synthetic promoters, $1 \times 1$ and $3 \times 1$, are statistically compared to the truncated-pCYC1core (two-sided Welch's t-test, see below and Table S1). C) Comparison of circuit 1 performance in the presence of a pre-crRNA and a single mature crRNA. Star symbols on top of a bar indicate a statistically significant difference between the relative fluorescence induced, in that circuit configuration, by the mature crRNA and the pre-crRNA ( $p$ values were calculated via two-sided Welch's t-test, see below and Table S3.2). D) crRNA expression from multicopy plasmids. Four circuit 1 configurations, based on the action of dLbCas12a-VPR on the $1 \times 1$ synthetic promoter, were modified by expressing the crRNA from multicopy plasmids. No circuit appeared to work properly. 1x1-dLb-VPR-RGRm-bA/bS did not show a statistically significant difference between the ON and the OFF state. 1x1-dLb-VPRpSNR52m-bA/bS even returned a relative fluorescence lower than 1 (see Table S2). E) dMbCas12a-based activators. We constructed eight circuits hosting dMbCas12a-VPR/VP64. Each of them expresses the crRNA via the pSNR52i cassette. Six circuits did not show any statistically significant difference between the ON and the OFF state. The working of the remaining two circuits cannot be assessed properly since their cells did not grow well in the presence of glucose. ns on top of a bar in figure $D$ and $E$ indicate a no statistically significant difference (two-sided Welch's t-test) between the ON and the OFF circuit configuration.

(“*”, p-value <0.05; “**”, p-value <0.01; “***”, p-value <0.001; “****”, p-value <0.0001; "ns”, no statistical difference). Each relative fluorescence level is the mean value obtained from at least 3 independent experiments i.e. carried out in different days. Error bars represent the standard deviation of the mean. 


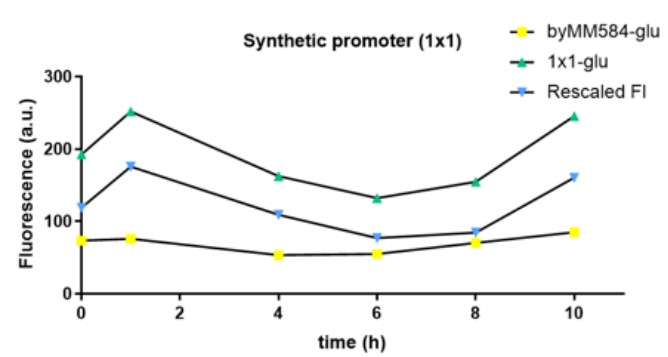

C

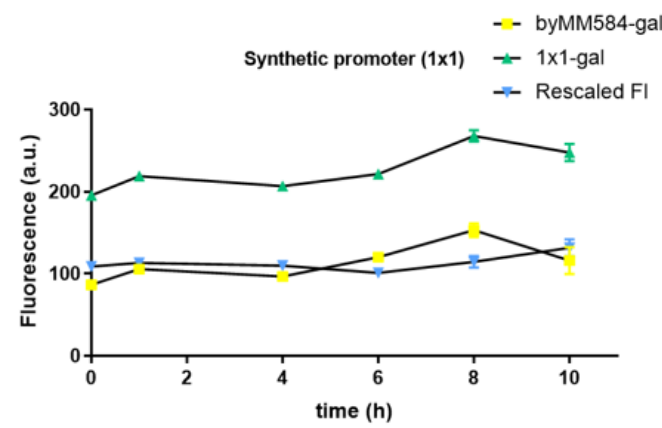

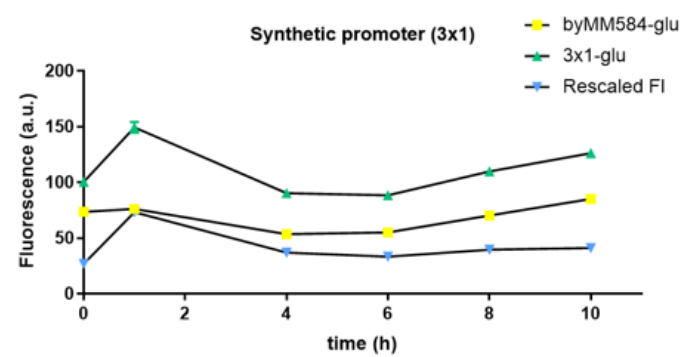

D

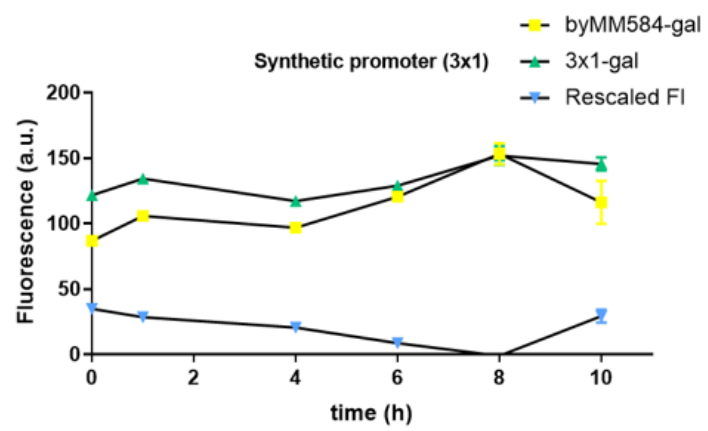

Figure S3. Monitoring synthetic promoter basal fluorescence expression over a 10-hour time period. Strain byMM555 (containing promoter configuration "1x1") and byMM556 ("3x1") were grown until reaching their steady state (14 hours, time $=0$ in the graphs) before starting the fluorescence intensity $(\mathrm{FI})$ measurements via FACS. Both strains were grown in glucose- (A and $\mathrm{B}$ ) and galactose-containing media ( $\mathrm{C}$ and $\mathrm{D}$ ). Rescaled $\mathrm{FI}$ (blue inverted triangles) is obtained by subtracting the background fluorescence (corresponding to byMM584-yellow squares) from the measured one (green triangle). Though not perfectly constant, the fluorescence emission from the four promoter is reasonably stable around its average value (A: 121.01 AU; B: $41.81 \mathrm{AU}$; C: $113.30 \mathrm{AU}$; D: $20.07 \mathrm{AU}$ ). Therefore, leakage effects can be excluded. Each data point represents the main value of three replicas. 


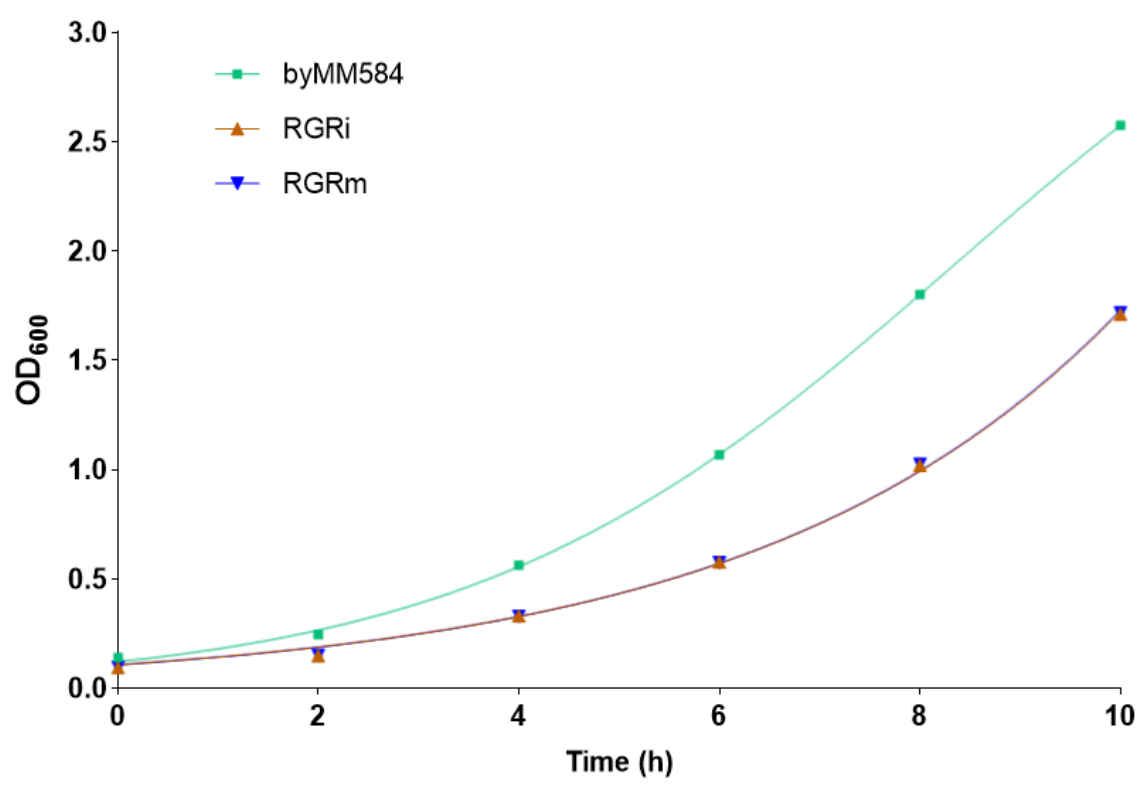

Figure S4. Impact of crRNA amount on $S$. cerevisiae cell fitness. We selected two variants of sub-circuit 2 (see Figure 2), namely: "1x1-dLb-VPR-RGRi-bA" and "1x1-dLb-VPR-RGRm-bA" in order to evaluate any effects on cell fitness induced by the amount of crRNA. Both strains produce crRNA via the RGR cassette that was placed either on an integrative (RGRi) or a multicopy (RGRm) plasmid. The growth of both strains was monitored over 10 hours and did not show any significative difference. Hence, we can conclude that the quantity of crRNA does not alter the fitness of $S$. cerevisiae cells. The growth slowdown of the two engineered strains with respect to the original chassis (byMM584) is probably imputable to the presence of the strong VPR activation domain fused to dLbCas12a. 


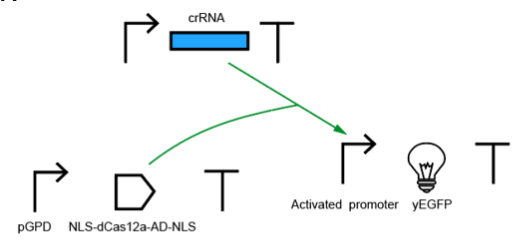

B

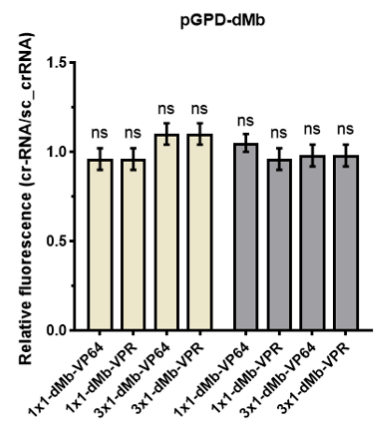

C

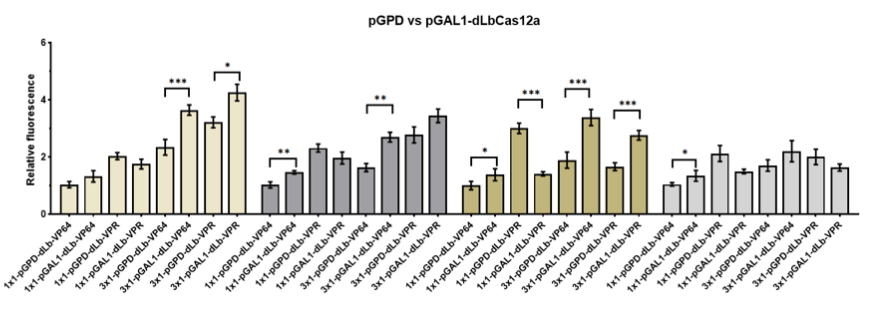

口pSNR52i-bA ש pSNR52i-bS ש RGRi-bA ص RGRi-bS

D
pGPD vs PGAL1-denAsCas12a

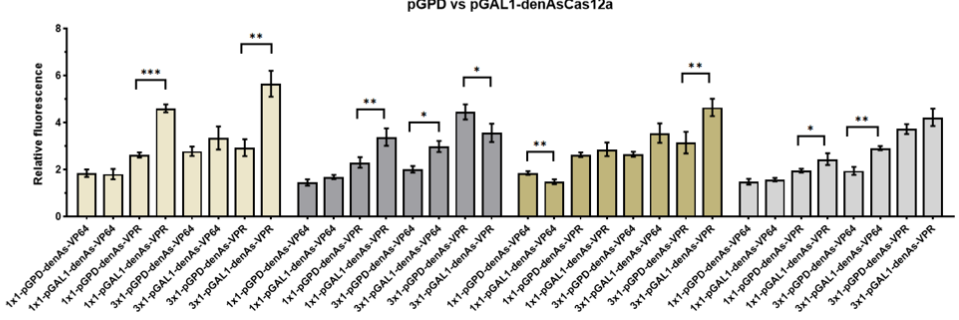

Figure S5. Three-gene sub-circuits from circuit 2. A) Sub-circuit scheme. Differently from the YES gates in Figure 1, dCas12a-AD is placed downstream of the constitutive GPD promoter. B) Relative fluorescence in the presence of dMbCas12a-AD. Each sub-circuit is labelled with the activated promoter configuration followed by "dMb" and the activation domain fused to $\mathrm{dMbCas12a}$. Different colors indicate different crRNA expression systems (see legend in C). CD) Comparison of the relative fluorescence expressed by sub-circuits 2 and their corresponding circuit 1 configurations. Each (sub-)circuit is specified by the number of operators in the synthetic promoter, the promoter expressing dCas12a-AD ( $\mathrm{GGPD}$ or pGAL1), an abbreviation of the name of the dCas12a protein, and the activation domain. Different colors correspond to different crRNA expression systems (see the legend in $\mathrm{C}$ ). Among the sub-circuits hosting dLbCas12a-ADs, only 1x1-pGPD-dLb-VPR-RGRi-bA showed a higher relative fluorescence than its corresponding YES gate. As for denAsCas12a-ADs, two sub-circuits (3x1-pGPDdenAs-VPR-pSNR52i-bS and 1x1-pGPD-denAs-RGRi-bA) performed better activation than their galactose-induced counterparts.

Star symbols on top of two adjacent bars indicate that there is a statistically significant difference between the two circuits. p-values were calculated via two-sided Welch's t-test ("*”, p-value <0.05; “**”, p-value <0.01; “***”, p-value <0.001). 
A

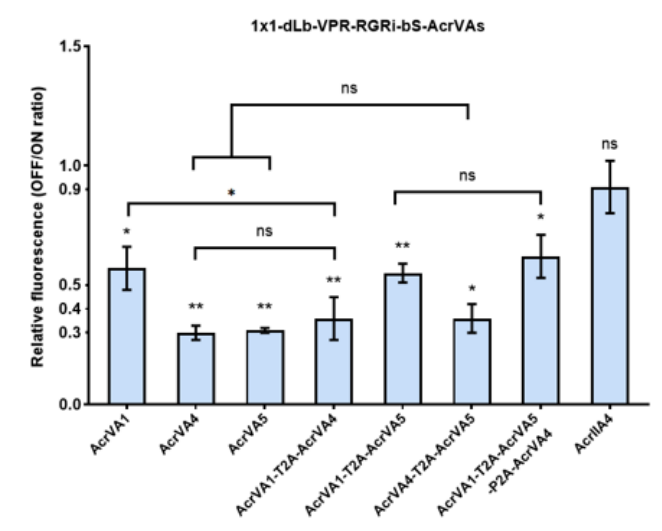

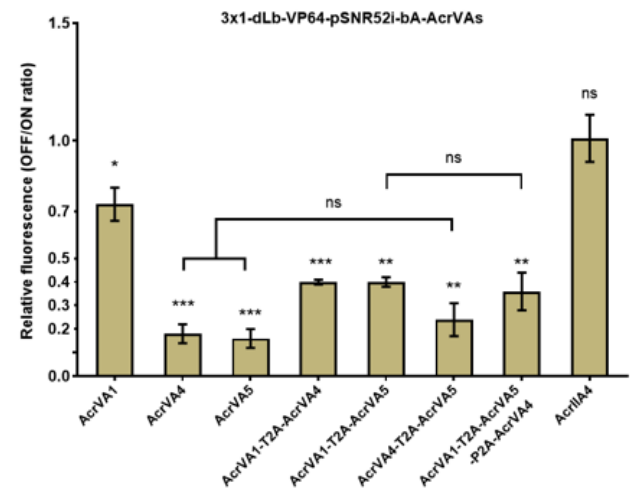

Figure S6. Co-expression of multiple AcrVAs. A) Inhibitory effects of single and multiple type V-A anti-CRISPR proteins on dLbCas12a-VPR. Expression of AcrVA1 together with AcrVA4 resulted in a decrease of the relative fluorescence associated with AcrVA1 alone. Other combinations of the anti-CRISPR proteins did not enhance the fluorescence inhibition by AcrVA4 and AcrVA5 alone. B) Inhibitory effects of single and multiple type V-A anti-CRISPR proteins on dLbCas12a-VP64. AcrVA1 alone performed poorly in this circuit. Co-expression of AcrVA1 with AcrVA4 and/or AcrVA5 resulted in more performant NOT gates. Nevertheless, the highest fluorescence reduction was achieved via either AcrVA4 or AcrVA5 alone. A detailed comparison of the circuits in A and B is given in Table S7.

Star symbols on top of a bar indicate that there is a statistically significant difference between circuit OFF and ON state. Star symbols on top of lines joining two bars indicate that there is a statistically significant difference between two circuits. $p$-values were calculated via two-sided Welch's t-test (“*”, p-value $<0.05$; “**”, p-value $<0.01$; “***”, p-value $<0.001$ ). 


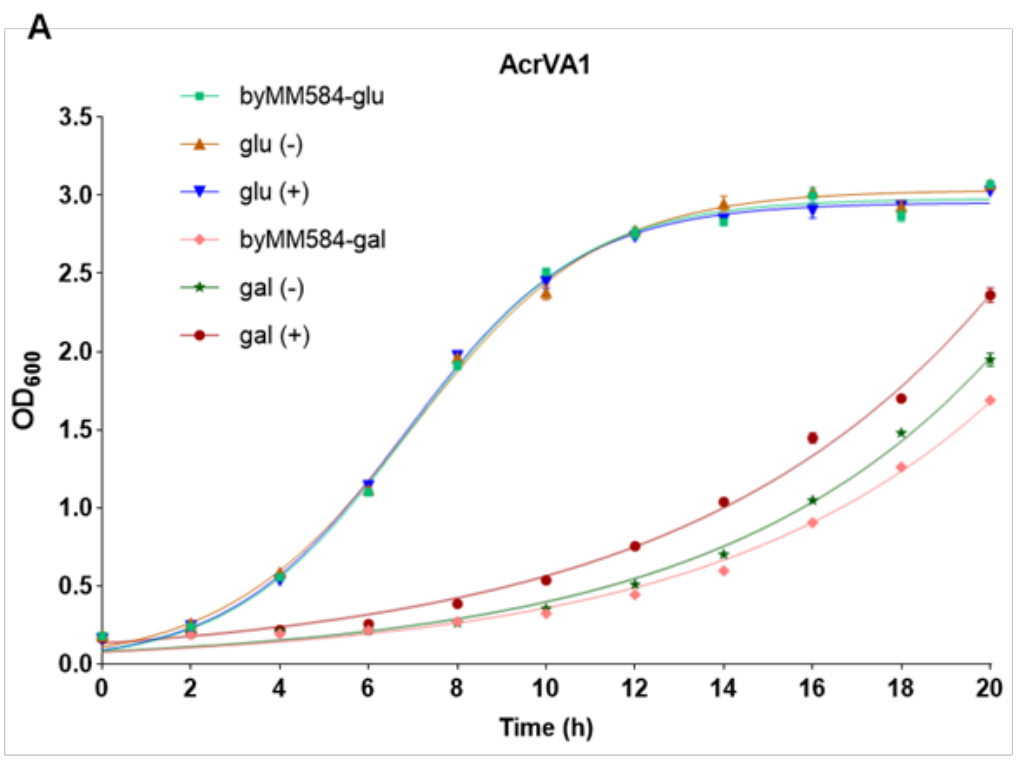

B

AcrVA4

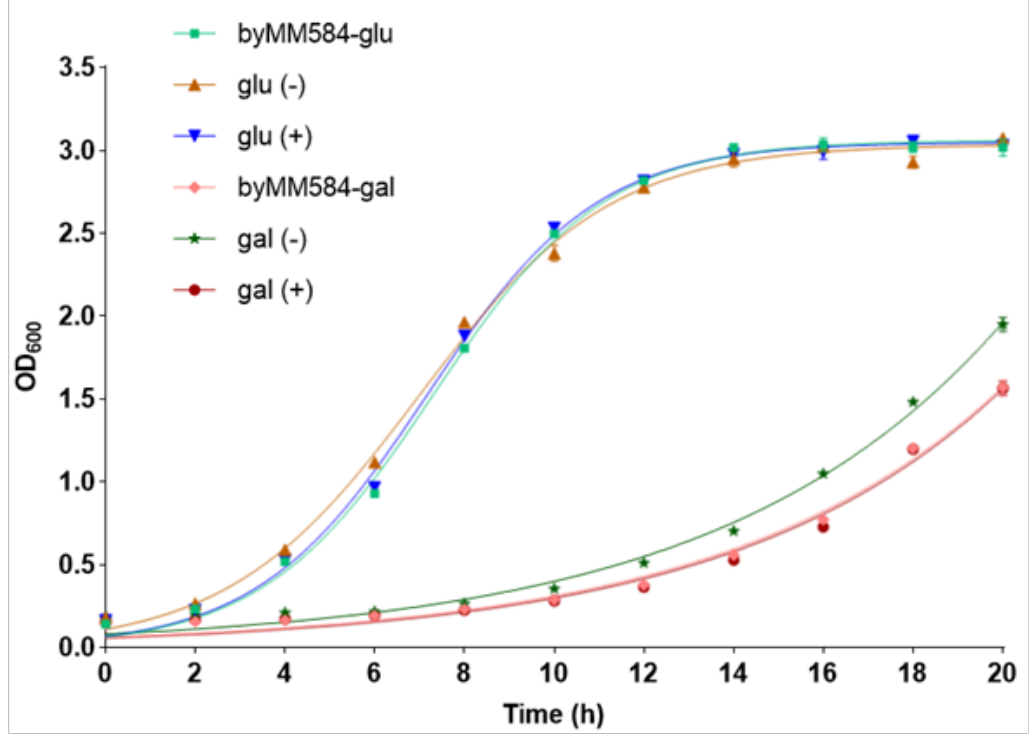

C

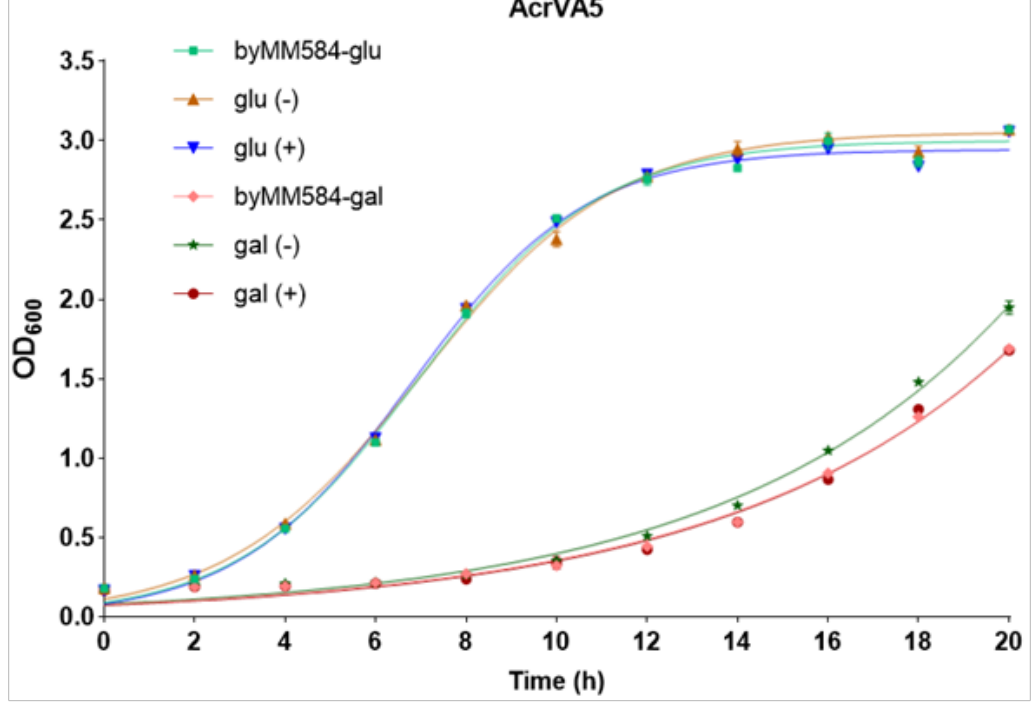


Figure S7. Impact of AcrVAs on S. cerevisiae cells fitness. The growth of strains engineered with circuit 3 (see Figure 3 ) i.e. containing the bare dLbCas12a, crRNA and AcrVA1 (A), AcrVA4 (B), or AcrVA5 (C) was monitored over a period of 20 hours. The symbols (+) indicates the presence of the AcrVA, whereas (-) signals its absence (i.e. the cells do not contain the whole circuit 3 since the TU encoding for the AcrVA is missing). As a further control, the original chassis (byMM584) was analyzed as well. Cells were grown in SDC supplemented with either $2 \%$ glucose or $2 \%$ galactose. None of the three AcrVAs appears to modify significantly the growth of yeast cells. Each data point represents the main value from three replicas.

A

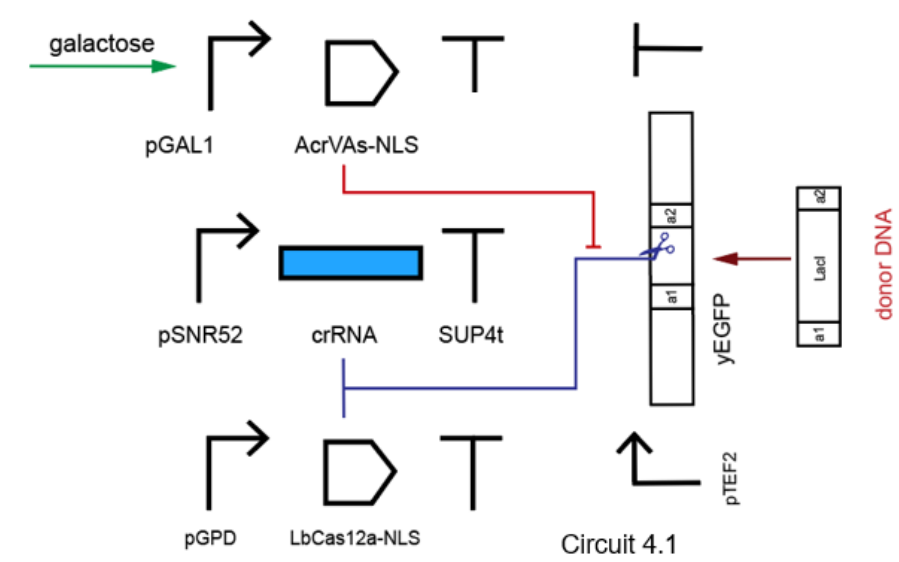

B

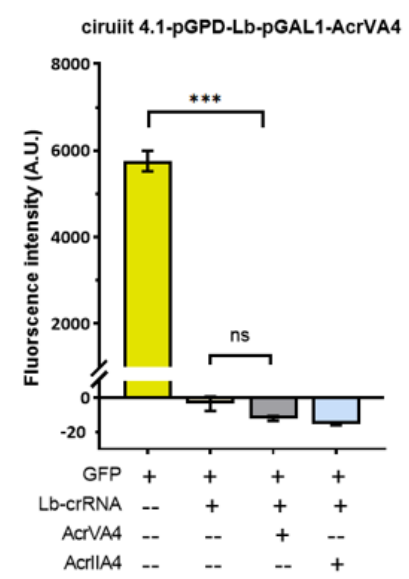

Figure S8 Gene editing circuit 4.1 A) Circuit scheme. LbCas12a is constitutively expressed by pGPD, AcrVA4 is placed downstream of pGAL1. B) The fluorescence intensity decreased to background level due to the cleavage of the $y E G F P$ gene by LbCas12a:crRNA and could not be restored by expressing AcrVA4. Statistical analysis was made via two-sided Welch's t-test. ( “***”, p-value <0.001). 
A

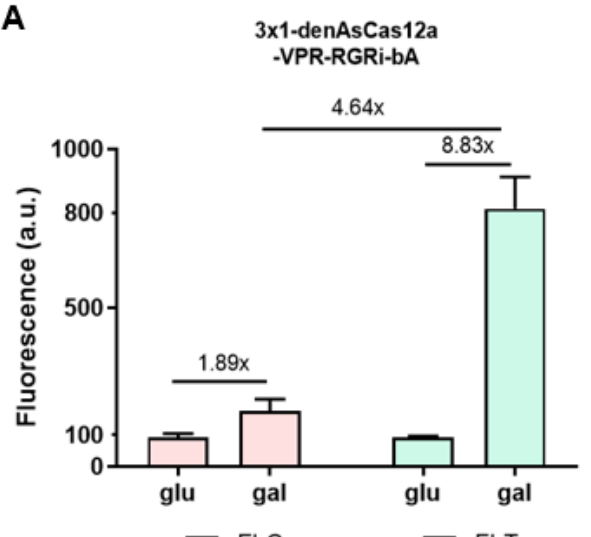

C

3x1-dCas12a-VPR

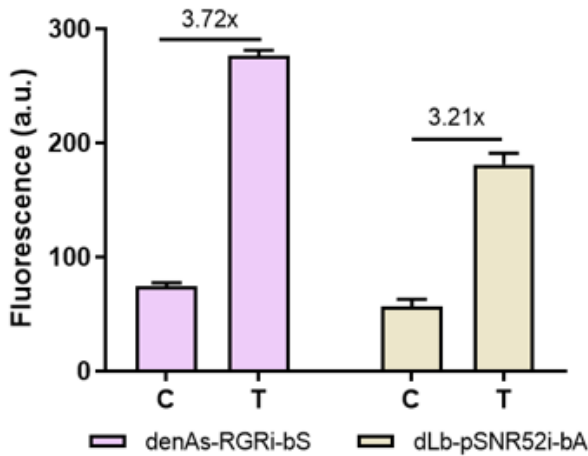

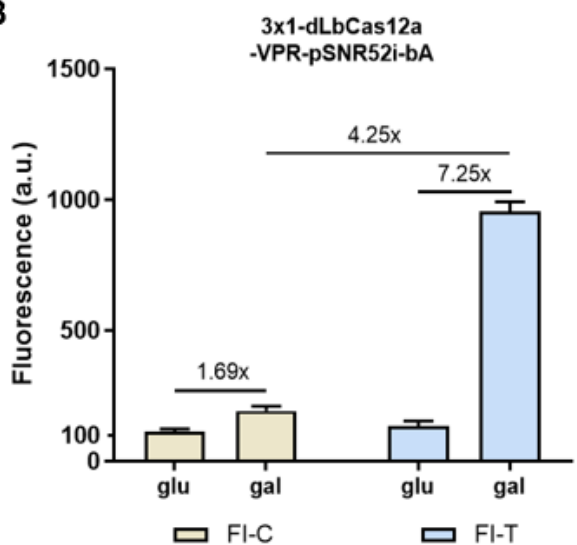

D

3×1-dLbCas12a-VP64 -pSNR52i-bA-AcrVA4

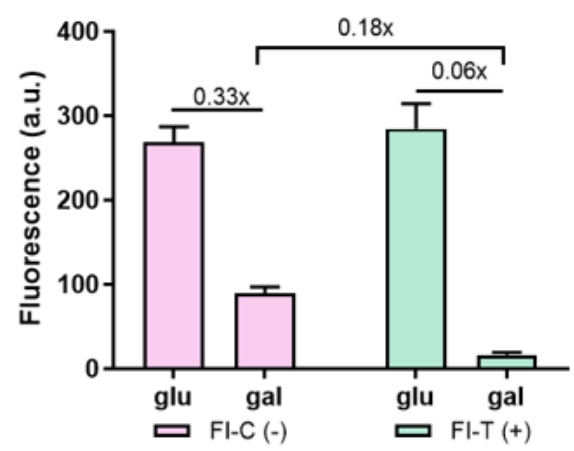

Figure S9. Absolute fluorescence intensities. A) According to Eq. (1) in "Materials and Methods", circuit 1 configuration " $3 \times 1-d A s-V P R-R G R i-b A$ " is associated with an ON/OFF ratio equal to 4.64-as reported on the top of the Figure. This value is obtained after measuring four fluorescence intensities: FI-T (where T stands for test i.e. the working circuit) in the presence of galactose $(\mathrm{ON} \mathrm{T})$ and glucose $(\mathrm{OFF})$; and $\mathrm{FI}-\mathrm{C}(\mathrm{C}=$ control), i.e. the basal fluorescence from the control circuit-in which a scrambled crRNA is expressed-also in the presence of glucose $\left(\mathrm{C}_{\mathrm{glu}}\right)$ and galactose $\left(\mathrm{C}_{\mathrm{gal}}\right)$. Eq. (1) avoids overestimating the circuit performance as $\mathrm{ON}_{\mathrm{T}} / \mathrm{OFF}_{\mathrm{T}}=8.83$ by requiring its division by the relative basal fluorescence $\mathrm{C}_{\text {gal }} / \mathrm{C}_{\text {glu }}$, which is higher than one (1.89) and, as such, has a negative influence on the gain in fluorescence due to the change of sugar in the cell solutions. B) Similar considerations hold for circuit 1 configuration "3x1-dLb-VPR-pSNR52i-bA". C) Fluorescence from two configurations of subcircuit 2 (see Figure 2A). Each sub-circuit is made of three TUs, none of them containing pGAL1. Hence, fluorescence from both test and control (hosting a scramble crRNA) is measured only in glucose-containing solutions. The ratio between the absolute values of the two fluorescence intensities is the ON/OFF ratio of the sub-circuits. D) The circuit 2 configuration " $3 \times 1-d L b-V P 64-$ pSNR52i-bA-A4" let register a relative fluorescence equal to 0.18 , which indicates a strong inhibition of AcrVA4 on dLbCas12a-VP64:crRNA. However, the action of AcrVA4 would be highly overestimated $\left(\mathrm{OFF}_{\mathrm{T}} / \mathrm{ON}_{\mathrm{T}}=0.06 ;(+)\right.$ indicates the presence of AcrVA4) if the decrease in the basal fluorescence in the presence of galactose had been neglected $\left(C_{\text {gal }} / C_{\text {glu }}=0.33 ;(-)\right.$ means the absence of AcrVA4).

Overall, relative fluorescence calculated via Eq. (1) permits both a proper estimation and a 
compact graphical representation (a single bar instead of four) of the performance of our circuits.

\section{Supplementary Tables}

Table S1. Mean fluorescence intensity generated by the promoters used in this work. SD is the standard deviation of the mean. The fluorescence intensity of pGAL1 was measured from cells grown in synthetic complete medium supplemented with $2 \%$ galactose. The p-value is calculated via two-sided Welch's t-test to compare $1 \times 1$ and $3 \times 1$ with the truncated-pCYC1core.

\begin{tabular}{lllll}
\hline Promoter-compare & $\begin{array}{l}\text { Mean fluorescence } \\
\text { intensity }\end{array}$ & SD & p-value & Replicates \\
\hline pGPD & 18390.48 & 1210.51 & 3 \\
pGAL1 & 16963.46 & 465.92 & 3 \\
pCYC1core & 1553.96 & 33.07 & 3 \\
truncated-pCYC1core & 110.47 & 6.07 & & 3 \\
1x1 & 154.36 & 9.46 & 0.0053 & 3 \\
$3 \times 1$ & 108.8 & 1.05 & 0.7201 & 3 \\
PTEF1 & 2741.91 & 238.05 & & 3 \\
PTEF2 & 8063.26 & 56.68 & & 3 \\
genCYC1t-pCYC1noTATA & 1023.17 & 58.71 & & 3 \\
\hline
\end{tabular}

Table S2. ON/OFF ratio--see Eq. (1) in the main text-corresponding to the circuit 1 variants built in this work. The mean fluorescence intensity $(\mathrm{FI})$ ON/OFF ratios are calculated on, at least, three independent experiments. SD represents the standard deviation of the mean. The p-value is calculated via two-sided Welch's t-test. Test circuits express the mature crRNA, whereas control circuits express a scrambled crRNA. The circuit is in ON state in the presence of galactose and OFF state in the presence of glucose.

\begin{tabular}{lllll}
\hline Circuit1: YES gate & Mean ON/OFF & SD & p-value & Replicates \\
\hline 1×1-dLb-VP64-pSNR52i-bA & 1.32 & 0.20 & 0.0142 & 4 \\
1×1-dLb-VP64-pSNR52i-bS & 1.46 & 0.06 & 0.0018 & 3 \\
1×1-dLb-VP64-RGRi-bA & 1.38 & 0.21 & 0.0038 & 4 \\
1×1-dLb-VP64-RGRi-bS & 1.34 & 0.91 & 0.0013 & 5 \\
1×1-dLb-VP64-pSNR52i-pre & 1.54 & 0.06 & 0.0415 & 3 \\
3x1-dLb-VP64-pSNR52i-bA & 3.64 & 0.18 & 0.0159 & 3 \\
3x1-dLb-VP64-pSNR52i-bS & 2.69 & 0.17 & 0.0004 & 3 \\
3x1-dLb-VP64-RGRi-bA & 3.38 & 0.28 & 0.0002 & 4 \\
3x1-dLb-VP64-RGRi-bS & 2.20 & 0.37 & 0.002 & 3 \\
3x1-dLb-VP64-pSNR52i-pre & 4.38 & 0.37 & 0.0105 & 3 \\
1x1-dLb-VPR-pSNR52i-bA & 1.75 & 0.17 & 0.0027 & 4 \\
1x1-dLb-VPR-pSNR52i-bS & 1.96 & 0.21 & $<0.0001$ & 4 \\
\hline
\end{tabular}




\begin{tabular}{|c|c|c|c|c|}
\hline 1x1-dLb-VPR-RGRi-bA & 1.41 & 0.07 & 0.0264 & 3 \\
\hline 1x1-dLb-VPR-RGRi-bS & 1.49 & 0.08 & 0.0001 & 3 \\
\hline 1x1-dLb-VPR-pSNR52i-pre & 2.56 & 0.12 & $<0.0001$ & 5 \\
\hline 3x1-dLb-VPR-pSNR52i-bA & 4.25 & 0.29 & 0.0037 & 3 \\
\hline 3x1-dLb-VPR-pSNR52i-bS & 3.44 & 0.24 & 0.0038 & 3 \\
\hline 3x1-dLb-VPR-RGRi-bA & 2.76 & 0.17 & $<0.0001$ & 3 \\
\hline 3×1-dLb-VPR-RGRi-bS & 1.63 & 0.12 & $<0.0001$ & 4 \\
\hline 3x1-dLb-VPR-pSNR52i-pre & 4.54 & 0.23 & 0.0014 & 4 \\
\hline 1x1-denAs-VP64-pSNR52i-bA & 1.8 & 0.22 & 0.0376 & 3 \\
\hline 1x1-denAs-VP64-pSNR52i-bS & 1.68 & 0.09 & 0.0152 & 3 \\
\hline 1x1-denAs-VP64-RGRi-bA & 1.48 & 0.10 & 0.0283 & 3 \\
\hline 1x1-denAs-VP64-RGRi-bS & 1.57 & 0.07 & 0.0064 & 3 \\
\hline 3x1-denAs-VP64-pSNR52i-bA & 3.34 & 0.49 & 0.0004 & 3 \\
\hline 3x1-denAs-VP64-pSNR52i-bS & 2.98 & 0.24 & 0.0001 & 3 \\
\hline 3x1-denAs-VP64-RGRi-bA & 3.55 & 0.41 & 0.0002 & 3 \\
\hline 3x1-denAs-VP64-RGRi-bS & 2.91 & 0.09 & 0.0002 & 4 \\
\hline 1x1-denAs-VPR-pSNR52i-bA & 4.60 & 0.17 & 0.0141 & 3 \\
\hline 1x1-denAs-VPR-pSNR52i-bS & 3.38 & 0.37 & 0.0006 & 4 \\
\hline 1x1-denAs-VPR-RGRi-bA & 2.86 & 0.29 & 0.0047 & 3 \\
\hline 1x1-denAs-VPR-RGRi-bS & 2.44 & 0.25 & 0.0009 & 4 \\
\hline 3x1-denAs-VPR-pSNR52i-bA & 5.65 & 0.55 & 0.0002 & 4 \\
\hline 3x1-denAs-VPR-pSNR52i-bS & 3.56 & 0.39 & $<0.0001$ & 4 \\
\hline 3x1-denAs-VPR-RGRi-bA & 4.64 & 0.37 & $<0.0001$ & 4 \\
\hline 3x1-denAs-VPR-RGRi-bS & 4.22 & 0.37 & 0.0001 & 5 \\
\hline 1x1-dMb-VP64-pSNR52i-bA & 1.00 & 0.15 & 0.9474 & 4 \\
\hline 1x1-dMb-VP64-pSNR52i-bS & 0.91 & 0.03 & 0.3473 & 4 \\
\hline 3x1-dMb-VP64-pSNR52i-bA & 0.93 & 0.27 & 0.5278 & 4 \\
\hline 3x1-dMb-VP64-pSNR52i-bS & 0.83 & 0.25 & 0.3047 & 3 \\
\hline 1x1-dMb-VPR-pSNR52i-bA & 1.94 & 0.11 & 0.0001 & 3 \\
\hline 1x1-dMb-VPR-pSNR52i-bS & 1.81 & 0.14 & $<0.0001$ & 4 \\
\hline 3x1-dMb-VPR-pSNR52i-bA & 1.02 & 0.17 & 0.9587 & 5 \\
\hline 3x1-dMb-VPR-pSNR52i-bS & 1.14 & 0.15 & 0.0975 & 4 \\
\hline 1x1-dLb-VPR-pSNR52m-bA & 0.78 & 0.09 & 0.0347 & 3 \\
\hline 1x1-dLb-VPR-pSNR52m-bS & 0.69 & 0.05 & 0.0189 & 3 \\
\hline 1x1-dLb-VPR-RGRm-bA & 0.95 & 0.06 & 0.4989 & 3 \\
\hline 1x1-dLb-VPR-RGRm-bS & 1.01 & 0.13 & 0.9347 & 3 \\
\hline
\end{tabular}

Tables S3. Comparison of the average relative fluorescence (mean ON/OFF ratio) measured on different circuit 1 configurations. SD represents the standard deviation of the mean. The $p$ value is calculated via two-sided Welch's t-test.

Table S3.1. Circuit 1: bA and bS in comparison. 


\begin{tabular}{|c|c|c|c|c|}
\hline pGAL1-dLb/denAs & Mean ON/OFF & SD & p-value & Replicates \\
\hline 1×1-dLb-VP64-pSNR52i-bA & 1.32 & 0.20 & & 4 \\
\hline 1x1-dLb-VP64-pSNR52i-bS & 1.46 & 0.06 & 0.3254 & 3 \\
\hline 1×1-dLb-VP64-RGRi-bA & 1.38 & 0.21 & & 4 \\
\hline 1x1-dLb-VP64-RGRi-bS & 1.34 & 0.91 & 0.9541 & 5 \\
\hline 1x1-dLb-VPR-pSNR52i-bA & 1.75 & 0.17 & & 4 \\
\hline 1x1-dLb-VPR-pSNR52i-bS & 1.96 & 0.21 & 0.2334 & 4 \\
\hline 1x1-dLb-VPR-RGRi-bA & 1.41 & 0.07 & & 3 \\
\hline 1×1-dLb-VPR-RGRi-bS & 1.49 & 0.08 & 0.3835 & 3 \\
\hline 3x1-dLb-VP64-pSNR52i-bA & 3.64 & 0.18 & & 3 \\
\hline 3x1-dLb-VP64-pSNR52i-bS & 2.69 & 0.17 & 0.0055 & 3 \\
\hline 3×1-dLb-VP64-RGRi-bA & 3.38 & 0.28 & & 4 \\
\hline 3×1-dLb-VP64-RGRi-bS & 2.20 & 0.37 & 0.0256 & 3 \\
\hline 3x1-dLb-VPR-pSNR52i-bA & 4.25 & 0.29 & & 3 \\
\hline 3x1-dLb-VPR-pSNR52i-bS & 3.44 & 0.24 & 0.0391 & 3 \\
\hline 3x1-dLb-VPR-RGRi-bA & 2.76 & 0.17 & & 3 \\
\hline 3×1-dLb-VPR-RGRi-bS & 1.63 & 0.12 & 0.0024 & 4 \\
\hline 1x1-denAs-VP64-pSNR52i-bA & 1.8 & 0.22 & & 3 \\
\hline 1x1-denAs-VP64-pSNR52i-bS & 1.68 & 0.09 & 0.5263 & 3 \\
\hline 1x1-denAs-VP64-RGRi-bA & 1.48 & 0.10 & & 3 \\
\hline 1x1-denAs-VP64-RGRi-bS & 1.57 & 0.07 & 0.3151 & 3 \\
\hline 1x1-denAs-VPR-pSNR52i-bA & 4.60 & 0.17 & & 3 \\
\hline 1x1-denAs-VPR-pSNR52i-bS & 3.38 & 0.37 & 0.0054 & 4 \\
\hline 1x1-denAs-VPR-RGRi-bA & 2.86 & 0.29 & & 3 \\
\hline 1x1-denAs-VPR-RGRi-bS & 2.44 & 0.25 & 0.1671 & 4 \\
\hline 3x1-denAs-VP64-pSNR52i-bA & 3.34 & 0.49 & & 3 \\
\hline 3x1-denAs-VP64-pSNR52i-bS & 2.98 & 0.24 & 0.4249 & 3 \\
\hline 3x1-denAs-VP64-RGRi-bA & 3.55 & 0.41 & & 3 \\
\hline 3x1-denAs-VP64-RGRi-bS & 2.91 & 0.09 & 0.9848 & 4 \\
\hline 3x1-denAs-VPR-pSNR52i-bA & 5.65 & 0.55 & & 4 \\
\hline 3x1-denAs-VPR-pSNR52i-bS & 3.56 & 0.39 & 0.0025 & 4 \\
\hline 3x1-denAs-VPR-RGRi-bA & 4.64 & 0.37 & & 4 \\
\hline 3×1-denAs-VPR-RGRi-bS & 4.22 & 0.37 & 0.1732 & 5 \\
\hline
\end{tabular}

Table S3.2. Circuit 1: pre-crRNA and single mature crRNA in comparison.

\begin{tabular}{lllll}
\hline pGAL1-dLb & Mean ON/OFF & SD & p-value & Replicates \\
\hline 1x1-dLb-VP64-pSNR52i-pre & 1.54 & 0.06 & & 3 \\
1x1-dLb-VP64-pSNR52i-bA & 1.32 & 0.20 & 0.1612 & 4 \\
1x1-dLb-VP64-pSNR52i-bS & 1.46 & 0.06 & 0.2841 & 3 \\
1x1-dLb-VP64-RGRi-bA & 1.38 & 0.21 & 0.2698 & 4 \\
1x1-dLb-VP64-RGRi-bS & 1.34 & 0.91 & 0.1064 & 5 \\
\hline 1x1-dLb-VPR-pSNR52i-pre & $\mathbf{2 . 5 6}$ & $\mathbf{0 . 1 2}$ & & $\mathbf{5}$ \\
\hline
\end{tabular}




\begin{tabular}{lllll}
\hline 1x1-dLb-VPR-pSNR52i-bA & 1.75 & 0.17 & 0.0007 & 4 \\
1x1-dLb-VPR-pSNR52i-bS & 1.96 & 0.21 & 0.0081 & 4 \\
1x1-dLb-VPR-RGRi-bA & 1.41 & 0.07 & $<0.0001$ & 3 \\
1x1-dLb-VPR-RGRi-bS & 1.49 & 0.08 & $<0.0001$ & 3 \\
\hline 3x1-dLb-VP64-pSNR52i-pre & 4.38 & 0.37 & & 3 \\
3x1-dLb-VP64-pSNR52i-bA & 3.64 & 0.18 & 0.0893 & 3 \\
3x1-dLb-VP64-pSNR52i-bS & 2.69 & 0.17 & 0.0121 & 3 \\
3x1-dLb-VP64-RGRi-bA & 3.38 & 0.28 & 0.0382 & 4 \\
3x1-dLb-VP64-RGRi-bS & 2.20 & 0.37 & 0.0043 & 3 \\
\hline 3x1-dLb-VPR-pSNR52i-pre & 4.54 & 0.23 & & 4 \\
3x1-dLb-VPR-pSNR52i-bA & 4.25 & 0.29 & 0.3097 & 3 \\
3x1-dLb-VPR-pSNR52i-bS & 3.44 & 0.24 & 0.0061 & 3 \\
3x1-dLb-VPR-RGRi-bA & 2.76 & 0.17 & 0.0002 & 3 \\
3x1-dLb-VPR-RGRi-bS & 1.63 & 0.12 & $<0.0001$ & 4 \\
\hline
\end{tabular}

Table S3.3. Circuit 1: pSNR52i and RGRi in comparison.

\begin{tabular}{lllll}
\hline pGAL1-dLb & Mean ON/OFF & SD & p-value & Replicates \\
\hline 1x1-dLb-VP64-pSNR52i-bA & 1.32 & 0.20 & & 4 \\
1x1-dLb-VP64-RGRi-bA & 1.38 & 0.21 & 0.7644 & 4 \\
\hline 1x1-dLb-VP64-pSNR52i-bS & 1.46 & 0.06 & & 3 \\
1x1-dLb-VP64-RGRi-bS & 1.34 & 0.91 & 0.2795 & 5 \\
\hline 1x1-dLb-VPR-pSNR52i-bA & 1.75 & 0.17 & & 4 \\
1x1-dLb-VPR-RGRi-bA & 1.41 & 0.07 & 0.0307 & 3 \\
\hline 1x1-dLb-VPR-pSNR52i-bS & 1.96 & 0.21 & & 4 \\
1x1-dLb-VPR-RGRi-bS & 1.49 & 0.08 & 0.0217 & 3 \\
\hline 3x1-dLb-VP64-pSNR52i-bA & 3.64 & 0.18 & & 3 \\
3x1-dLb-VP64-RGRi-bA & 3.38 & 0.28 & 0.2413 & 4 \\
\hline 3x1-dLb-VP64-pSNR52i-bS & 2.69 & 0.17 & & 3 \\
3x1-dLb-VP64-RGRi-bS & 2.20 & 0.37 & 0.1966 & 3 \\
\hline 3x1-dLb-VPR-pSNR52i-bA & 4.25 & 0.29 & & 3 \\
3x1-dLb-VPR-RGRi-bA & 2.76 & 0.17 & 0.0064 & 3 \\
\hline 3x1-dLb-VPR-pSNR52i-bS & 3.44 & 0.24 & & 3 \\
3x1-dLb-VPR-RGRi-bS & 1.63 & 0.12 & 0.0033 & 4 \\
\hline 1x1-denAs-VP64-pSNR52i-bA & 1.8 & 0.22 & & 3 \\
1x1-denAs-VP64-RGRi-bA & 1.48 & 0.10 & 0.1569 & 3 \\
\hline 1x1-denAs-VP64-pSNR52i-bS & 1.68 & 0.09 & & 3 \\
1x1-denAs-VP64-RGRi-bS & 1.57 & 0.07 & 0.2167 & 3 \\
\hline 1x1-denAs-VPR-pSNR52i-bA & 4.60 & 0.17 & & 3 \\
1x1-denAs-VPR-RGRi-bA & 2.86 & 0.29 & 0.0003 & 3 \\
\hline 1x1-denAs-VPR-pSNR52i-bS & 3.38 & 0.37 & & 4 \\
1x1-denAs-VPR-RGRi-bS & 2.44 & 0.0137 & 4 \\
\hline
\end{tabular}




\begin{tabular}{lllll}
\hline 3x1-denAs-VP64-pSNR52i-bA & 3.34 & 0.49 & & 3 \\
3x1-denAs-VP64-RGRi-bA & 3.55 & 0.41 & 0.6627 & 3 \\
\hline 3x1-denAs-VP64-pSNR52i-bS & 2.98 & 0.24 & & 3 \\
3x1-denAs-VP64-RGRi-bS & 2.91 & 0.09 & 0.7133 & 4 \\
\hline 3×1-denAs-VPR-pSNR52i-bA & 5.65 & 0.55 & & 4 \\
3x1-denAs-VPR-RGRi-bA & 4.64 & 0.37 & 0.046 & 4 \\
\hline 3×1-denAs-VPR-pSNR52i-bS & 3.56 & 0.39 & & 4 \\
3x1-denAs-VPR-RGRi-bS & 4.22 & 0.37 & 0.0653 & 5 \\
\hline
\end{tabular}

Table S4. Mean relative fluorescence (ON/OFF) expressed by different sub-circuit 2 configurations. SD represents the standard deviation of the mean. The $p$-value is calculated via two-sided Welch's t-test.

\begin{tabular}{|c|c|c|c|c|}
\hline Circuit 2: sub-circuit & Mean ON/OFF & SD & $p$-value & Replicates \\
\hline 1x1-denAs-VP64-pSNR52i-bA & 1.84 & 0.16 & 0.0038 & 4 \\
\hline 1x1-denAs-VP64-pSNR52i-bS & 1.45 & 0.13 & 0.0371 & 3 \\
\hline 1x1-denAs-VP64-RGRi-bA & 1.85 & 0.08 & 0.0017 & 4 \\
\hline 1x1-denAs-VP64-RGRi-bS & 1.48 & 0.12 & 0.0197 & 4 \\
\hline 3×1-denAs-VP64-pSNR52i-bA & 2.78 & 0.20 & 0.004 & 3 \\
\hline 3x1-denAs-VP64-pSNR52i-bS & 2.01 & 0.14 & 0.0038 & 3 \\
\hline 3x1-denAs-VP64-RGRi-bA & 2.65 & 0.11 & 0.0098 & 3 \\
\hline 3x1-denAs-VP64-RGRi-bS & 1.94 & 0.17 & 0.0023 & 3 \\
\hline 1x1-denAs-VPR-pSNR52i-bA & 2.62 & 0.11 & 0.0001 & 4 \\
\hline 1x1-denAs-VPR-pSNR52i-bS & 2.30 & 0.22 & 0.0008 & 3 \\
\hline 1x1-denAs-VPR-RGRi-bA & 2.63 & 0.10 & $<0.0001$ & 5 \\
\hline 1x1-denAs-VPR-RGRi-bS & 1.95 & 0.08 & $<0.0001$ & 5 \\
\hline 3x1-denAs-VPR-pSNR52i-bA & 2.93 & 0.36 & 0.0091 & 3 \\
\hline 3×1-denAs-VPR-pSNR52i-bS & 4.45 & 0.32 & 0.0012 & 4 \\
\hline 3x1-denAs-VPR-RGRi-bA & 3.14 & 0.46 & 0.0004 & 4 \\
\hline 3x1-denAs-VPR-RGRi-bS & 3.72 & 0.21 & $<0.0001$ & 3 \\
\hline 1x1-dLb-VP64-pSNR52i-bA & 1.03 & 0.11 & 0.8203 & 3 \\
\hline 1x1-dLb-VP64-pSNR52i-bS & 1.02 & 0.11 & 0.8454 & 3 \\
\hline 1x1-dLb-VP64-RGRi-bA & 1.00 & 0.15 & 0.9505 & 3 \\
\hline 1x1-dLb-VP64-RGRi-bS & 1.04 & 0.06 & 0.6666 & 3 \\
\hline 3x1-dLb-VP64-pSNR52i-bA & 2.34 & 0.27 & 0.0001 & 5 \\
\hline 3x1-dLb-VP64-pSNR52i-bS & 1.63 & 0.14 & 0.0011 & 4 \\
\hline 3×1-dLb-VP64-RGRi-bA & 1.89 & 0.28 & 0.002 & 4 \\
\hline 3x1-dLb-VP64-RGRi-bS & 1.70 & 0.20 & 0.0008 & 4 \\
\hline 1x1-dLb-VPR-pSNR52i-bA & 2.03 & 0.12 & 0.0017 & 4 \\
\hline 1x1-dLb-VPR-pSNR52i-bS & 2.31 & 0.14 & 0.0003 & 5 \\
\hline 1x1-dLb-VPR-RGRi-bA & 3.00 & 0.18 & $<0.0001$ & 4 \\
\hline 1x1-dLb-VPR-RGRi-bS & 2.12 & 0.28 & 0.0218 & 3 \\
\hline 3x1-dLb-VPR-pSNR52i-bA & 3.21 & 0.19 & 0.0003 & 3 \\
\hline
\end{tabular}




\begin{tabular}{lllll}
\hline 3x1-dLb-VPR-pSNR52i-bS & 2.77 & 0.28 & 0.0005 & 3 \\
3x1-dLb-VPR-RGRi-bA & 1.66 & 0.14 & 0.0008 & 5 \\
3x1-dLb-VPR-RGRi-bS & 2.00 & 0.27 & 0.0361 & 3 \\
1x1-dMb-VP64-pSNR52i-bA & 0.96 & 0.06 & 0.5035 & 3 \\
1x1-dMb-VP64-pSNR52i-bS & 1.05 & 0.05 & 0.1863 & 3 \\
3x1-dMb-VP64-pSNR52i-bA & 1.10 & 0.06 & 0.1255 & 3 \\
3x1-dMb-VP64-pSNR52i-bS & 0.98 & 0.06 & 0.6554 & 3 \\
1x1-dMb-VPR-pSNR52i-bA & 1.01 & 0.08 & 0.8812 & 3 \\
1x1-dMb-VPR-pSNR52i-bS & 1.01 & 0.08 & 0.897 & 3 \\
3x1-dMb-VPR-pSNR52i-bA & 0.95 & 0.12 & 0.64 & 3 \\
3x1-dMb-VPR-pSNR52i-bS & 0.97 & 0.08 & 0.78 & 3 \\
1x1-dLb-VPR-pSNR52m-bA & 1.42 & 0.02 & 0.0166 & 3 \\
1x1-dLb-VPR-pSNR52m-bS & 1.48 & 0.09 & 0.0284 & 3 \\
1x1-dLb-VPR-RGRm-bA & 1.25 & 0.03 & 0.033 & 3 \\
1x1-dLb-VPR-RGRm-bS & 1.28 & 0.03 & 0.0445 & 2 \\
\hline
\end{tabular}

Table S5. Mean OFF/ON ratio from the NOT gates (circuit 2) built in this work. SD represents the standard deviation on the mean. The $p$-value is calculated via two-sided Welch's t-test. Test circuits express AcrVA, control circuits do not. The OFF state is realized by growing cells in the presence of galactose, the $\mathrm{ON}$ state demands, in contrast, glucose.

\begin{tabular}{lllll}
\hline Circuit 2: NOT gate & Mean OFF/ON & SD & p-value & Replicates \\
\hline 3x1-denAs-VP64-pSNR52i-bA-AcrVA1 & 0.81 & 0.06 & 0.0086 & 3 \\
3x1-denAs-VP64-RGRi-bA-AcrVA1 & 0.68 & 0.04 & 0.0029 & 3 \\
3x1-denAs-VP64-pSNR52i-bA-AcrlIA4 & 1.25 & 0.16 & 0.0812 & 3 \\
1x1-denAs-VPR-pSNR52i-bA-AcrVA1 & 0.64 & 0.05 & 0.0014 & 3 \\
3x1-denAs-VPR-pSNR52i-bS-AcrVA1 & 0.55 & 0.03 & 0.0494 & 3 \\
3x1-denAs-VPR-RGRi-bS-AcrVA1 & 0.29 & 0.10 & 0.0205 & 4 \\
1x1-denAs-VPR-pSNR52i-bA-AcrVA4 & 1.12 & 0.099 & 0.5757 & 3 \\
1x1-denAs-VPR-pSNR52i-bA-AcrVA5 & 1.04 & 0.11 & 0.8242 & 3 \\
1x1-denAs-VPR-pSNR52i-bA-AcrlIA4 & 1.27 & 0.12 & 0.1265 & 3 \\
3x1-dLb-VP64-pSNR52i-bA-AcrVA1 & 0.73 & 0.07 & 0.0114 & 3 \\
3x1-dLb-VP64-pSNR52i-bA-AcrVA4 & 0.18 & 0.04 & 0.0002 & 3 \\
3x1-dLb-VP64-pSNR52i-bA-AcrVA5 & 0.16 & 0.04 & 0.0002 & 3 \\
3x1-dLb-VP64-pSNR52i-bA-AcrlIA4 & 1.01 & 0.10 & 0.874 & 4 \\
3x1-dLb-VP64-RGRi-bA-AcrVA4 & 0.54 & 0.04 & 0.0056 & 3 \\
3x1-dLb-VP64-RGRi-bA-AcrIIA4 & 1.05 & 0.19 & 0.7871 & 3 \\
1x1-dLb-VPR-RGRi-bS-AcrVA1 & 0.57 & 0.09 & 0.0459 & 3 \\
1x1-dLb-VPR-RGRi-bS-AcrVA4 & 0.30 & 0.03 & 0.0094 & 3 \\
1x1-dLb-VPR-RGRi-bS-AcrVA5 & 0.31 & 0.01 & 0.0256 & 3 \\
1x1-dLb-VPR-RGRi-bS-AcrIIA4 & 0.91 & 0.11 & 0.5024 & 3 \\
1x1-dLb-VPR-pSNR52i-bA-AcrVA4 & 0.58 & 0.07 & 0.0133 & 3 \\
\hline
\end{tabular}




\begin{tabular}{lllll}
\hline 1×1-dLb-VPR-pSNR52i-bS-AcrVA4 & 0.48 & 0.14 & 0.0392 & 3 \\
3×1-dLb-VPR-pSNR52i-bA-AcrVA5 & 0.24 & 0.07 & 0.003 & 4 \\
3×1-dLb-VPR-pSNR52i-bS-AcrVA1 & 0.32 & 0.02 & 0.0169 & 3 \\
3×1-dLb-VPR-RGRi-bA-AcrVA1 & 0.16 & 0.04 & 0.0189 & 3 \\
3x1-dLb-VPR-RGRi-bA-AcrVA4 & 0.38 & 0.05 & 0.0003 & 3 \\
3x1-dLb-VPR-RGRi-bS-AcrVA1 & 0.23 & 0.06 & 0.013 & 3 \\
3x1-dLb-VPR-RGRi-bS-AcrVA4 & 0.24 & 0.05 & 0.0082 & 3 \\
\hline
\end{tabular}

Table S6. Mean OFF/ON ratio of circuit 2 upon co-expression of multiple AcrVAs. SD represents the standard deviation of the mean. The p-value is calculated via two-sided Welch's t-test, as explained in Table S5 above.

\begin{tabular}{lllll}
\hline Circuit 2-2A & Mean OFF/ON & SD & p-value & Replicates \\
\hline $\begin{array}{l}\text { 3x1-dLb-VP64-pSNR52i-bA-AcrVA1- } \\
\text { T2A-AcrVA4 }\end{array}$ & 0.40 & 0.01 & 0.0002 & 3 \\
$\begin{array}{l}\text { 3x1-dLb-VP64-pSNR52i-bA-AcrVA1- } \\
\text { T2A-AcrVA5 }\end{array}$ & 0.40 & 0.02 & 0.002 & 3 \\
$\begin{array}{l}\text { 3x1-dLb-VP64-pSNR52i-bA-AcrVA4- } \\
\text { T2A-AcrVA5 }\end{array}$ & 0.24 & 0.07 & 0.0045 & 3 \\
$\begin{array}{l}\text { 3x1-dLb-VP64-pSNR52i-bA-AcrVA1- } \\
\text { T2A-AcrVA5-P2A-AcrVA4 }\end{array}$ & 0.36 & 0.08 & 0.0033 & 3 \\
$\begin{array}{l}\text { 1x1-dLb-VPR-RGRi-bS-AcrVA1- } \\
\text { T2A-AcrVA4 }\end{array}$ & 0.36 & 0.09 & 0.0073 & 5 \\
$\begin{array}{l}\text { 1x1-dLb-VPR-RGRi-bS-AcrVA1- } \\
\text { T2A-AcrVA5 }\end{array}$ & 0.55 & 0.04 & 0.0052 & 4 \\
$\begin{array}{l}\text { 1x1-dLb-VPR-RGRi-bS-AcrVA4- } \\
\text { T2A-AcrVA5 }\end{array}$ & 0.36 & 0.06 & 0.0105 & 3 \\
$\begin{array}{l}\text { 1x1-dLb-VPR-RGRi-bS-AcrVA1- } \\
\text { T2A-AcrVA5-P2A-AcrVA4 }\end{array}$ & 0.62 & & & \\
\hline
\end{tabular}

Table S7. Comparison of circuit 2 average relative fluorescence (mean OFF/ON ratio) achieved by the only AcrVA1 and multiple AcrVAs. SD represents the standard deviation of the mean. The p-value is calculated via two-sided Welch's t-test.

\begin{tabular}{lllll}
\hline Circuit 2-2A & Mean OFF/ON & SD & p-value & Replicates \\
\hline 3x1-dLb-VP64-pSNR52i-bA-AcrVA1- & $\mathbf{0 . 4 0}$ & $\mathbf{0 . 0 1}$ & & 3 \\
T2A-AcrVA4 & & & & \\
3x1-dLb-VP64-pSNR52i-bA-AcrVA1 & 0.73 & 0.07 & 0.0239 & 3 \\
3x1-dLb-VP64-pSNR52i-bA-AcrVA4 & 0.18 & 0.04 & 0.0101 & 3 \\
\hline 3x1-dLb-VP64-pSNR52i-bA-AcrVA1- & $\mathbf{0 . 4 0}$ & $\mathbf{0 . 0 2}$ & & 3 \\
T2A-AcrVA5 & & & & \\
\hline
\end{tabular}




\begin{tabular}{lllll}
\hline 3x1-dLb-VP64-pSNR52i-bA-AcrVA1 & 0.73 & 0.07 & 0.0205 & 3 \\
3x1-dLb-VP64-pSNR52i-bA-AcrVA5 & 0.16 & 0.04 & 0.0039 & 3 \\
\hline 3x1-dLb-VP64-pSNR52i-bA-AcrVA4- & $\mathbf{0 . 2 4}$ & $\mathbf{0 . 0 7}$ & & 3 \\
T2A-AcrVA5 & & & & \\
3x1-dLb-VP64-pSNR52i-bA-AcrVA4 & 0.18 & 0.04 & 0.32 & 3 \\
3x1-dLb-VP64-pSNR52i-bA-AcrVA5 & 0.16 & 0.04 & 0.2169 & 3 \\
\hline 3x1-dLb-VP64-pSNR52i-bA-AcrVA1- & $\mathbf{0 . 3 6}$ & $\mathbf{0 . 0 8}$ & & $\mathbf{3}$ \\
T2A-AcrVA5-P2A-AcrVA4 & & & & \\
3x1-dLb-VP64-pSNR52i-bA-AcrVA1 & 0.73 & 0.07 & 0.0091 & 3 \\
3x1-dLb-VP64-pSNR52i-bA-AcrVA5 & 0.16 & 0.04 & 0.0486 & 3 \\
3x1-dLb-VP64-pSNR52i-bA-AcrVA4 & 0.18 & 0.04 & 0.0614 & 3 \\
3x1-dLb-VP64-pSNR52i-bA-AcrVA1- & 0.40 & 0.02 & 0.5217 & 3 \\
T2A-AcrVA5 & & & & \\
\hline 1x1-dLb-VPR-RGRi-bS-AcrVA1- & $\mathbf{0 . 3 6}$ & $\mathbf{0 . 0 9}$ & & $\mathbf{5}$ \\
T2A-AcrVA4 & & & & \\
1x1-dLb-VPR-RGRi-bS-AcrVA1 & 0.57 & 0.09 & 0.0442 & 3 \\
1x1-dLb-VPR-RGRi-bS-AcrVA4 & 0.30 & 0.03 & 0.3122 & 3 \\
\hline 1x1-dLb-VPR-RGRi-bS-AcrVA1- & $\mathbf{0 . 5 5}$ & $\mathbf{0 . 0 4}$ & & $\mathbf{4}$ \\
T2A-AcrVA5 & & & & 3 \\
1x1-dLb-VPR-RGRi-bS-AcrVA1 & 0.57 & 0.09 & 0.8055 & 3 \\
1x1-dLb-VPR-RGRi-bS-AcrVA5 & 0.31 & 0.01 & 0.0009 & 3 \\
\hline 1x1-dLb-VPR-RGRi-bS-AcrVA4- & $\mathbf{0 . 3 6}$ & $\mathbf{0 . 0 6}$ & & $\mathbf{3}$ \\
T2A-AcrVA5 & & & & 3 \\
1x1-dLb-VPR-RGRi-bS-AcrVA4 & 0.30 & 0.03 & 0.2771 & 3 \\
1x1-dLb-VPR-RGRi-bS-AcrVA5 & 0.31 & 0.01 & 0.3425 & 3 \\
\hline 1x1-dLb-VPR-RGRi-bS-AcrVA1- & $\mathbf{0 . 6 2}$ & $\mathbf{0 . 0 9}$ & & $\mathbf{4}$ \\
T2A-AcrVA5-P2A-AcrVA4 & & & & 3 \\
1x1-dLb-VPR-RGRi-bS-AcrVA1 & 0.57 & 0.09 & 0.5585 & 3 \\
1x1-dLb-VPR-RGRi-bS-AcrVA5 & 0.31 & 0.01 & 0.005 & 3 \\
1x1-dLb-VPR-RGRi-bS-AcrVA4 & 0.30 & 0.03 & 0.0087 & 3 \\
1x1-dLb-VPR-RGRi-bS-AcrVA1- & 0.55 & 0.04 & 0.2906 & 4 \\
T2A-AcrVA5 & & & & \\
\hline
\end{tabular}

Table S8. Mean relative fluorescence corresponding to three-gene sub-circuits obtained from circuit 3. SD represented the standard deviation of the mean. The $p$-value is calculated via twosided Welch's t-test.

\begin{tabular}{lllll}
\hline Sub-circuit & $\begin{array}{l}\text { Mean relative fluorescence } \\
\text { (crRNA/no_crRNA) }\end{array}$ & SD & p-value & Replicates \\
\hline GFP-denAs-crRNA & 0.31 & 0.03 & 0.0005 & 4 \\
GFP-dLb-crRNA & 0.55 & 0.06 & 0.0018 & 4 \\
GFP-dMb-crRNA & 0.97 & 0.02 & 0.8872 & 3 \\
\hline
\end{tabular}


Table S9. Mean ON/OFF ratio of circuits 3, calculated as explained in Table S5. SD represents the standard deviation of the mean. The p-value is calculated via two-sided Welch's t-test.

\begin{tabular}{lllll}
\hline pGAL1-AcrVAs & Mean ON/OFF & SD & p-value & Replicates \\
\hline GFP-denAs-crRNA-AcrVA1 & 1.63 & 0.06 & 0.0063 & 3 \\
GFP-denAs-crRNA-AcrlIA4 & 1.05 & 0.13 & 0.6321 & 3 \\
GFP-dLb-crRNA-AcrVA1 & 1.81 & 0.07 & 0.0002 & 3 \\
GFP-dLb-crRNA-AcrVA4 & 1.89 & 0.17 & 0.0072 & 3 \\
GFP-dLb-crRNA-AcrVA5 & 1.57 & 0.02 & $<0.0001$ & 5 \\
GFP-dLb-crRNA-AcrllA4 & 1.03 & 0.11 & 0.7279 & 3 \\
\hline
\end{tabular}

Table S10. Mean relative fluorescence in circuit 3 when AcrVAs are expressed by promoters of different strength. SD represents the standard deviation of the mean. The $p$-value is calculated via two-sided Welch's t-test to assess if there is a statistically significant difference between test and control circuit (the latter without AcrVAs).

\begin{tabular}{lllll}
\hline pTEF1-AcrVAs & Mean relative fluorescence & SD & p-value & Replicates \\
\hline GFP-denAs-crRNA-AcrVA1 & 1.08 & 0.06 & 0.1802 & 3 \\
GFP-dLb-crRNA-AcrVA1 & 1.36 & 0.03 & 0.0061 & 5 \\
GFP-dLb-crRNA-AcrVA4 & 1.97 & 0.08 & $<0.0001$ & 4 \\
GFP-dLb-crRNA-AcrVA5 & 1.69 & 0.11 & 0.0219 & 3 \\
\hline
\end{tabular}

\begin{tabular}{lllll}
\hline genCYC1t-pCYC1noTATA-AcrVAs & $\begin{array}{l}\text { Mean relative } \\
\text { fluorescence }\end{array}$ & SD & p-value & Replicates \\
\hline GFP-denAs-crRNA-AcrVA1 & 1.06 & 0.05 & 0.6524 & 4 \\
GFP-dLb-crRNA-AcrVA1 & 1.19 & 0.04 & 0.007 & 3 \\
GFP-dLb-crRNA-AcrVA4 & 1.80 & 0.04 & 0.0016 & 3 \\
GFP-dLb-crRNA-AcrVA5 & 1.70 & 0.03 & $<0.0001$ & 3 \\
\hline
\end{tabular}

Table S11. Mean relative fluorescence in circuits 2 (circuit configurations: 1x1-denAs-VPRpSNR52i-bA and 1x1-dLb-VPR-RGRi-bS) when the AcrVAs are expressed by promoters of different strength. SD represents the standard deviation of the mean. The $p$-value is calculated via two-sided Welch's t-test to assess if there is statistically significant difference between test and control circuit (the latter without AcrVAs).

\begin{tabular}{lllll}
\hline \multirow{2}{*}{ pTEF1-AcrVAs } & $\begin{array}{l}\text { Mean relative } \\
\text { fluorescence }\end{array}$ & SD & p-value & Replicates \\
\hline 1x1-denAs-VPR-pSNR52i-bA-AcrVA1 & 0.99 & 0.05 & 0.8822 & 3 \\
1x1-dLb-VPR-RGRi-bS-AcrVA1 & 1.08 & 0.06 & 0.3415 & 4 \\
\hline
\end{tabular}




\begin{tabular}{lllll}
\hline 1x1-dLb-VPR-RGRi-bS-AcrVA4 & 0.57 & 0.02 & 0.0013 & 3 \\
1x1-dLb-VPR-RGRi-bS-AcrVA5 & 0.54 & 0.04 & 0.0015 & 4 \\
\hline
\end{tabular}

\begin{tabular}{lllll}
\hline genCYC1t-pCYC1noTATA-AcrVAs & $\begin{array}{l}\text { Mean relative } \\
\text { fluorescence }\end{array}$ & SD & p-value & Replicates \\
\hline 1x1-denAs-VPR-pSNR52i-bA-AcrVA1 & 1.02 & 0.055 & 0.9222 & 4 \\
1x1-dLb-VPR-RGRi-bS-AcrVA1 & 1.05 & 0.01 & 0.1499 & 4 \\
1x1-dLb-VPR-RGRi-bS-AcrVA4 & 0.55 & 0.005 & 0.0007 & 3 \\
1x1-dLb-VPR-RGRi-bS-AcrVA5 & 0.53 & 0.02 & 0.0003 & 3 \\
\hline
\end{tabular}

Table S12. Comparison of circuit 2 and circuit 3 mean relative fluorescence achieved by expressing AcrVAs with different promoters. SD represents the standard deviation of the mean. The $p$-value is calculated via two-sided Welch's t-test.

\begin{tabular}{lllll}
\hline Circuit 2-dLb & $\begin{array}{l}\text { Mean relative } \\
\text { fluorescence }\end{array}$ & SD & p-value & Replicates \\
\hline pGAL1-AcrVA1 & $\mathbf{0 . 5 7}$ & $\mathbf{0 . 0 9}$ & 3 \\
pTEF1-AcrVA1 & 1.08 & 0.06 & 0.0038 & 4 \\
genCYC1t-pCYC1noTATA -AcrVA1 & 1.05 & 0.01 & 0.0151 & 4 \\
\hline pTEF1-AcrVA1 & $\mathbf{1 . 0 8}$ & $\mathbf{0 . 0 6}$ & & 4 \\
genCYC1t-pCYC1noTATA -AcrVA1 & 1.05 & 0.01 & 0.5268 & 4 \\
\hline pGAL1-AcrVA4 & $\mathbf{0 . 3 0}$ & $\mathbf{0 . 0 3}$ & & 3 \\
pTEF1-AcrVA4 & 0.57 & 0.02 & 0.0011 & 3 \\
genCYC1t-pCYC1noTATA -AcrVA4 & 0.55 & 0.005 & 0.0041 & 3 \\
\hline pTEF1-AcrVA4 & $\mathbf{0 . 5 7}$ & $\mathbf{0 . 0 2}$ & & 3 \\
genCYC1t-pCYC1noTATA -AcrVA4 & 0.55 & 0.005 & 0.6515 & 3 \\
\hline pGAL1-AcrVA5 & $\mathbf{0 . 3 1}$ & $\mathbf{0 . 0 1}$ & & 3 \\
pTEF1-AcrVA5 & 0.54 & 0.04 & 0.002 & 4 \\
genCYC1t-pCYC1noTATA -AcrVA5 & 0.53 & 0.02 & 0.0036 & 3 \\
\hline pTEF1-AcrVA5 & $\mathbf{0 . 5 4}$ & $\mathbf{0 . 0 4}$ & & 4 \\
genCYC1t-pCYC1noTATA -AcrVA5 & 0.53 & 0.02 & 0.5911 & 3 \\
\hline
\end{tabular}

\begin{tabular}{lllll}
\hline Circuit 3-dLb & $\begin{array}{l}\text { Mean relative } \\
\text { fluorescence }\end{array}$ & SD & p-value & Replicates \\
\hline pGAL1-AcrVA1 & $\mathbf{1 . 8 1}$ & $\mathbf{0 . 0 7}$ & & 3 \\
pTEF1-AcrVA1 & 1.36 & 0.03 & 0.0071 & 5 \\
genCYC1t-pCYC1noTATA -AcrVA1 & 1.19 & 0.04 & 0.0015 & 3 \\
\hline pTEF1-AcrVA1 & $\mathbf{1 . 3 6}$ & $\mathbf{0 . 0 3}$ & & \\
genCYC1t-pCYC1noTATA -AcrVA1 & 1.19 & 0.04 & 0.0174 & \\
\hline
\end{tabular}




\begin{tabular}{lllll}
\hline pGAL1-AcrVA4 & $\mathbf{1 . 8 9}$ & $\mathbf{0 . 1 7}$ & & 3 \\
pTEF1-AcrVA4 & 1.97 & 0.08 & 0.8832 & 4 \\
genCYC1t-pCYC1noTATA -AcrVA4 & 1.69 & 0.11 & 0.5217 & 3 \\
\hline pTEF1-AcrVA4 & $\mathbf{1 . 9 7}$ & $\mathbf{0 . 0 8}$ & & \\
genCYC1t-pCYC1noTATA -AcrVA4 & 1.69 & 0.11 & 0.055 & \\
\hline pGAL1-AcrVA5 & $\mathbf{1 . 5 7}$ & $\mathbf{0 . 0 2}$ & 5 \\
pTEF1-AcrVA5 & 1.69 & 0.11 & 0.2681 & 3 \\
genCYC1t-pCYC1noTATA -AcrVA5 & 1.70 & 0.03 & 0.0134 & 3 \\
\hline pTEF1-AcrVA5 & $\mathbf{1 . 6 9}$ & $\mathbf{0 . 1 1}$ & & \\
genCYC1t-pCYC1noTATA -AcrVA5 & 1.70 & 0.03 & 0.9121 & \\
\hline
\end{tabular}

Table S13. Mean fluorescence intensity from circuits 4 , in which both LbCas12a and AcrVA4 are expressed under pGPD or pGAL1. Each mean value is obtained from three independent experiments. SD represents the standard deviation of the mean.

\begin{tabular}{lll}
\hline $\begin{array}{l}\text { Circuit 4.1 } \\
\text { pGPD-Lb-pGAL1-AcrVA4 }\end{array}$ & $\begin{array}{l}\text { Mean fluorescence } \\
\text { intensity }\end{array}$ & SD \\
\hline GFP & 5755.49 & 236.26 \\
GFP-Lb-crRNA & -3.4 & 4.16 \\
GFP-Lb-crRNA-AcrVA4 & -12.03 & 1.38 \\
GFP-Lb-crRNA-AcrlIA4 & -15.14 & 0.83 \\
\hline
\end{tabular}

\begin{tabular}{lll}
\hline $\begin{array}{l}\text { Circuit 4.2 } \\
\text { pGAL1-Lb-pGPD-AcrVA4 }\end{array}$ & $\begin{array}{l}\text { Mean fluorescence } \\
\text { intensity }\end{array}$ & SD \\
\hline GFP & 6285.73 & 127.36 \\
GFP-Lb-crRNA & -4.62 & 3.66 \\
GFP-Lb-crRNA-AcrVA4 & 4147.61 & 396.54 \\
GFP-Lb-crRNA-AcrlIA4 & 14.73 & 7.42 \\
\hline
\end{tabular}

\begin{tabular}{lll}
\hline $\begin{array}{l}\text { Circuit 4.3 } \\
\text { pGPD-Lb-pGPD-AcrVA4 }\end{array}$ & $\begin{array}{l}\text { Mean fluorescence } \\
\text { intensity }\end{array}$ & SD \\
\hline GFP & 8063.26 & 56.68 \\
GFP-Lb-crRNA & -3.40 & 4.16 \\
GFP-Lb-crRNA-AcrVA4 & 3335.62 & 243.21 \\
GFP-Lb-crRNA-AcrlIA4 & -0.45 & 6.18 \\
\hline
\end{tabular}


Table S14. Plasmids constructed in this work (the first five were bought from Addgene).

\begin{tabular}{|c|c|}
\hline Plasmid name & Content \\
\hline pRSII403 & Addgene-35436 \\
\hline pRSII404 & Addgene-35438 \\
\hline pRSII405 & Addgene-35440 \\
\hline pRSII406 & Addgene-35442 \\
\hline pRSII424 & Addgene-35466 \\
\hline pMM724 & pRSII405-lexOpR-truncated-pCYC1core-yEGFP-Tsynth24 \\
\hline pMM729 & pRSII405-3xlexOpR-truncated-pCYC1core-yEGFP-Tsynth24 \\
\hline pMM736 & $\begin{array}{l}\text { pRSII406-pGAL1-ATG-NLS-GS-HIStag-GS-BamHI-sp-Xhol- } \\
\text { linker_yo_VPR_NLS-TAA-mTGuo1 }\end{array}$ \\
\hline pMM751 & pRSII404-pSNR52-DR(MbCas12a)_spacer (lexOpR,20nt, bA)-SUP4t \\
\hline pMM752 & pRSII424-pSNR52-DR(MbCas12a)_spacer (lexOpR,20nt, bA)-SUP4t \\
\hline pMM755 & pRSII404-pSNR52-DR(LbCas12a)_spacer (lexOpR,20nt, bA)-SUP4t \\
\hline pMM756 & pRSII424-pSNR52-DR(LbCas12a)_spacer (lexOpR,20nt, bA)-SUP4t \\
\hline pMM759 & pRSII404-pSNR52-DR(LbCas12a)_spacer (lexOpR,21nt, bS)-SUP4t \\
\hline pMM760 & pRSII424-pSNR52-DR(LbCas12a)_spacer (lexOpR,21nt, bS)-SUP4t \\
\hline pMM761 & pRSII404-pSNR52-DR(MbCas12a)_spacer (lexOpR,21nt, bS)-SUP4t \\
\hline pMM762 & pRSII424-pSNR52-DR(MbCas12a)_spacer (lexOpR,21nt, bS)-SUP4 \\
\hline pMM763 & pRSII406-pGAL1-yEGFP-mTGUO1 \\
\hline pMM764 & $\begin{array}{l}\text { pRSII403-pGAL1-ATG-NLS-GS-HIStag-GS-BamHI-sp-Xhol-GS-NLS-TAA- } \\
\text { CYC1t }\end{array}$ \\
\hline pMM766 & $\begin{array}{l}\text { pRSII403-pGAL1-ATG-FLAGtag-GS-BamHI-sp-Xhol-NLS-GS-NLS-TAA- } \\
\text { Tsynth6 }\end{array}$ \\
\hline pMM774 & $\begin{array}{l}\text { pRSII406-pGAL1-ATG-NLS-GS-HIStag-GS-BamHI-dLbCas12a } \\
\text {-Xhol- linker_yo_VPR_NLS-TAA-mTGuo1 }\end{array}$ \\
\hline pMM776 & $\begin{array}{l}\text { pRSII406-pGAL1-ATG-NLS-GS-HIStag-GS-BamHI-denAsCas12a } \\
\text {-Xhol- linker_yo_VPR_NLS-TAA-mTGuo1 }\end{array}$ \\
\hline pMM779 & pRSII404-pSNR52-DR(AsCas12a)_spacer (lexOpR,20nt, bA)-SUP4t \\
\hline pMM780 & pRSII424-pSNR52-DR(AsCas12a)_spacer (lexOpR,20nt, bA)-SUP4t \\
\hline
\end{tabular}




\begin{tabular}{|c|c|}
\hline pMM784 & $\begin{array}{l}\text { pRSII403-pGAL1-ATG-NLS-GS-HIStag-GS-BamHI-yo_AcrVA1-Xhol-NLS- } \\
\text { GS-NLS- TAA-Tsynth6 }\end{array}$ \\
\hline pMM790 & pRSII404-pSNR52-DR(AsCas12a)_spacer (lexOpR,21nt, bS)-SUP4t \\
\hline pMM791 & pRSII424-pSNR52-DR(AsCas12a)_spacer (lexOpR,21nt, bS)-SUP4t \\
\hline pMM814 & $\begin{array}{l}\text { pRSII406-pGPD-ATG-NLS-GS-HIStag-GS-BamHI-dLbCas12a-Xhol- } \\
\text { linker_yo_VPR_NLS-TAA-mTGuo1 }\end{array}$ \\
\hline pMM815 & $\begin{array}{l}\text { pRSII406-pGPD-ATG-NLS-GS-HIStag-GS-BamHI-denAsCas12a-Xhol- } \\
\text { linker_yo_VPR_NLS-TAA-mTGuo1 }\end{array}$ \\
\hline pMM818 & pRSII404-pADH1-RGR[DR(AsCas12a)_spacer (lexOpR,21nt, bS)]-ADH1t \\
\hline pMM819 & pRSII424-pADH1-RGR[DR(AsCas12a)_spacer (lexOpR,21nt, bS)]-ADH1t \\
\hline pMM820 & pRSII404-pADH1-RGR[DR(AsCas12a)_spacer (lexOpR,20nt, bA)]-ADH1t \\
\hline pMM830 & pRSII424-pADH1-RGR[DR(AsCas12a)_spacer (lexOpR,20nt, bA)]-ADH1t \\
\hline pMM834 & pRSII404-pADH1-RGR[DR(LbCas12a)_spacer (lexOpR,20nt, bS)]-ADH1t \\
\hline pMM835 & pRSII424-pADH1-RGR[DR(LbCas12a)_spacer (lexOpR,20nt, bS)]-ADH1t \\
\hline pMM836 & pRSII404-pADH1-RGR[DR(LbCas12a)_spacer (lexOpR,20nt, bA)]-ADH1t \\
\hline pMM837 & pRSII424-pADH1-RGR[DR(LbCas12a)_spacer (lexOpR,20nt, bA)]-ADH1t \\
\hline pMM838 & pRSII403-pTEF2-AcrVA1-GS-HBD(ER)-CYC1t \\
\hline pMM846 & $\begin{array}{l}\text { pRSII406-pGPD-ATG-NLS-GS-HIStag-GS-BamHI-dLbCas12a-Xhol } \\
\text {-linker_yoVP64_NLS-TAA-mTGuo1 }\end{array}$ \\
\hline pMM847 & $\begin{array}{l}\text { pRSII406-pGPD-ATG-NLS-GS-HIStag-GS-BamHI-denAsCas12a-Xhol- } \\
\text { linker_yoVP64_NLS-TAA-mTGuo1 }\end{array}$ \\
\hline pMM848 & $\begin{array}{l}\text { pRSII406-pGAL1-ATG-NLS-GS-HIStag-GS-BamHI-dLbCas12a-Xhol } \\
\text {-linker_yoVP64_NLS-TAA-mTGuo1 }\end{array}$ \\
\hline pMM849 & $\begin{array}{l}\text { pRSII406-pGAL1-ATG-NLS-GS-HIStag-GS-BamHI-denAsCas12a-Xhol- } \\
\text { linker_yoVP64_NLS-TAA-mTGuo1 }\end{array}$ \\
\hline pMM850 & pRSII404-pSNR52-DR (LbCas12a)_scrambled-spacer-SUP4t \\
\hline pMM851 & pRSII424-pSNR52-DR (LbCas12a)_scrambled-spacer-SUP4t \\
\hline pMM875 & $\begin{array}{l}\text { pRSII404-pSNR52-DR (dAsCas12a)_scrambled-spacer (lexOpR,20nt)- } \\
\text { SUP4t }\end{array}$ \\
\hline pMM877 & $\begin{array}{l}\text { pMM47-pGAL1-ATG-NLS-GS-HIStag-GS-BamHI-dLbCas12a-Xhol-GS- } \\
\text { NLS-TAA-CYC1t }\end{array}$ \\
\hline pMM878 & $\begin{array}{l}\text { pMM47-pGAL1-ATG-NLS-GS-HIStag-GS-BamHI-denAsCas12a-Xhol-GS- } \\
\text { NLS-TAA-CYC1t }\end{array}$ \\
\hline pMM879 & $\begin{array}{l}\text { pMM47-pGPD-ATG-NLS-GS-HIStag-GS-BamHI-dLbCas12a-Xhol-GS- } \\
\text { NLS-TAA-CYC1t }\end{array}$ \\
\hline pMM880 & $\begin{array}{l}\text { pMM47-pGPD-ATG-NLS-GS-HIStag-GS-BamHI-denAsCas12a-Xhol-GS- } \\
\text { NLS-TAA-CYC1t }\end{array}$ \\
\hline pMM882 & pRSII405-pSNR52-DR(LbCas12a)-sgRNA- SUP4t for gene editing-SUP4t \\
\hline pMM895 & $\begin{array}{l}\text { pRSII403-pGAL1-ATG-NLS-GS-HIStag-GS-BamHI-yo_AcrVA4-Xhol-GS- } \\
\text { NLS-TAA-CYC1t }\end{array}$ \\
\hline pMM898 & $\begin{array}{l}\text { pRSII406-pGAL1-ATG-NLS-GS-HIStag-GS-BamHI-LbCas12a-Xhol-GS- } \\
\text { NLS-TAA-CYC1t }\end{array}$ \\
\hline
\end{tabular}




\begin{tabular}{|c|c|}
\hline pMM913 & $\begin{array}{l}\text { pRSII406-pGPD-ATG-NLS-GS-HIStag-GS-BamHI-dMbCas12a-Xhol } \\
\text {-linker_yoVP64-NLS-TAA-mTGuo1 }\end{array}$ \\
\hline pMM914 & $\begin{array}{l}\text { pRSII406-pGAL1-ATG-NLS-GS-HIStag-GS-BamHI-dMbCas12a-Xhol } \\
\text {-linker_yoVP64 NLS-TAA-mTGuo1 }\end{array}$ \\
\hline pMM915 & $\begin{array}{l}\text { pMM47-pGPD-ATG-NLS-GS-HIStag-GS-BamHI-dMbCas12a-Xhol-GS- } \\
\text { NLS-TAA-CYC1t }\end{array}$ \\
\hline pMM916 & $\begin{array}{l}\text { pRSII406-pGAL1-ATG-NLS-GS-HIStag-GS-BamHI-dMbCas12a-Xhol } \\
\text {-linker_yo_VPR_NLS-TAA-mTGuo1 }\end{array}$ \\
\hline pMM917 & $\begin{array}{l}\text { pRSII403-pGAL1-ATG-NLS-GS-FLAGtag-GS-BamHI-yo_AcrVA1-T2A- } \\
\text { HA_tag-AcrVAA4-Xhol-GS-NLS-TAA-CYC1t }\end{array}$ \\
\hline pMM937 & $\begin{array}{l}\text { pRSII403-pGPD-ATG-NLS-GS-FLAGtag-GS-BamHI-yo_AcrVA4-Xhol-GS- } \\
\text { NLS-TAA-CYC1t }\end{array}$ \\
\hline pMM938 & pRSII405-pSNR52-DR(AsCas12a)-sgRNA- SUP4t for gene editing-SUP4t \\
\hline pMM939 & $\begin{array}{l}\text { pMM47-pGPD-ATG-NLS-GS-HIStag-GS-BamHI-dLbCas12a-Xhol-GS- } \\
\text { NLS-TAA-CYC1t }\end{array}$ \\
\hline pMM940 & pRSII405-pSNR52-DR(MbCas12a)-sgRNA- SUP4t for gene editing-SUP4t \\
\hline pMM941 & $\begin{array}{l}\text { pRSII403-pGAL1-ATG-NLS-GS-FLAGtag-GS-BamHI-yo_AcrVA5-Xhol- } \\
\text { GS-NLS-TAA-CYC1t }\end{array}$ \\
\hline pMM975 & $\begin{array}{l}\text { pRSII403-pGAL1-ATG-NLS-GS-FLAGtag_GS-BamHI-yo_AcrVA4-T2A- } \\
\text { HA-AcrVA5-Xhol-GS-NLS-TAA-CYC1t }\end{array}$ \\
\hline pMM976 & $\begin{array}{l}\text { pRSII406-pTEF1-ATG-NLS-GS-HIStag-GS-BamHI-dLbCas12a-Xhol- } \\
\text { linker_yoVP64_NLS-TAA-mTGuo1 }\end{array}$ \\
\hline pMM977 & $\begin{array}{l}\text { pRSII403-pGAL1-ATG-NLS-GS-FLAGtag-GS-BamHI-yo_AcrVA1-T2A-HA- } \\
\text { AcrVAA5-Xhol-GS-NLS-TAA-CYC1t }\end{array}$ \\
\hline pMM978 & $\begin{array}{l}\text { pRSII406-pGPD-ATG-NLS-GS-HIStag-GS-BamHI-dMbCas12a-Xhol- } \\
\text { linker_yo_VPR_NLS-TAA-mTGuo1 }\end{array}$ \\
\hline pMM986 & pMM404-pSNR52-DR(LbCas12a)-s1(bA)-DR(LbCas12a)-s2(bS)-SUP4t \\
\hline pMM987 & $\begin{array}{l}\text { pRSII403-pTEF1-ATG-NLS-GS-FLAGtag-GS-BamHI-yo_AcrVA5-Xhol- } \\
\text { GS-NLS-TAA-CYC1t }\end{array}$ \\
\hline pMM1005 & $\begin{array}{l}\text { pRSII406-pTEF1-ATG-NLS-GS-HIStag-GS-BamHI-dLbCas12a-Xhol- } \\
\text { linker_yo_VPR_NLS-TAA-mTGuo1 }\end{array}$ \\
\hline pMM1084 & $\begin{array}{l}\text { pRSII403-pGAL1-ATG-NLS-GS-FLAGtag_GS-BamHI-yo_AcrVA1-T2A- } \\
\text { HA-AcrVA5-P2A-Myctag-AcrVA4-Xhol-GS-NLS-TAA-CYC1t }\end{array}$ \\
\hline pMM1085 & $\begin{array}{l}\text { pRSII403-pTEF1-ATG-NLS-GS-FLAGtag-GS-BamHI-yo_AcrVA1-Xhol- } \\
\text { GS-NLS-TAA-CYC1t }\end{array}$ \\
\hline pMM1086 & $\begin{array}{l}\text { pRSII403-pTEF1-ATG-NLS-GS-FLAGtag-GS-BamHI-yo_AcrVA4-Xhol- } \\
\text { GS-NLS-TAA-CYC1t }\end{array}$ \\
\hline pMM1087 & $\begin{array}{l}\text { pRSII403-genCYC1t-pCYC1noTATA-ATG-NLS-GS-FLAGtag-GS-BamHI- } \\
\text { yo_AcrVA1-Xhol-GS-NLS-TAA-CYC1t }\end{array}$ \\
\hline pMM1088 & $\begin{array}{l}\text { pRSII403-genCYC1t-pCYC1noTATA-ATG-NLS-GS-FLAGtag-GS-BamHI- } \\
\text { yo_AcrVA4-Xhol-GS-NLS-TAA-CYC1t }\end{array}$ \\
\hline pMM1089 & $\begin{array}{l}\text { pRSII403-genCYC1t-pCYC1noTATA-ATG-NLS-GS-FLAGtag-GS-BamHI- } \\
\text { yo_AcrVA5-Xhol-GS-NLS-TAA-CYC1t }\end{array}$ \\
\hline
\end{tabular}


Table S15. Yeast strains engineered in this work (the first one was bought from Euroscarf).

\begin{tabular}{|c|c|}
\hline Strain name & Genotype \\
\hline byMM584 & $\begin{array}{l}\text { CEN.PK2-1C (MATa; his3D1; leu2-3 112; ura3-52; trp1-289; MAL2-8c; } \\
\text { SUC2) }\end{array}$ \\
\hline byMM555 & byMM584 pMM724:: Leu2 \\
\hline byMM556 & byMM584 pMM729:: Leu2 \\
\hline byMM571 & byMM584 pMM763:: Ura3 \\
\hline byMM575 & byMM584 pMM729:: Leu2 pMM776:: Ura3 \\
\hline byMM585 & byMM584 pMM724:: Leu2 pMM774:: Ura3 \\
\hline byMM586 & byMM584 pMM724:: Leu2 pMM776:: Ura3 \\
\hline byMM587 & byMM584 pMM729:: Leu2 pMM776:: Ura3 pMM779:: Trp1 \\
\hline byMM588 & byMM584 pMM729:: Leu2 pMM776:: Ura3 pMM790:: Trp1 \\
\hline byMM590 & byMM584 pMM724:: Leu2 pMM815:: Ura3 \\
\hline byMM591 & byMM584 pMM729:: Leu2 pMM815:: Ura3 \\
\hline byMM592 & byMM584 pMM724:: Leu2 pMM774:: Ura3 pMM755:: Trp1 \\
\hline byMM593 & byMM584 pMM724:: Leu2 pMM774:: Ura3 pMM759:: Trp1 \\
\hline byMM594 & byMM584 pMM724:: Leu2 pMM776:: Ura3 pMM779:: Trp1 \\
\hline byMM595 & byMM584 pMM724:: Leu2 pMM776:: Ura3 pMM790:: Trp1 \\
\hline byMM596 & byMM584 pMM724:: Leu2 pMM815:: Ura3 pMM779:: Trp1 \\
\hline byMM597 & byMM584 pMM724:: Leu2 pMM815:: Ura3 pMM790:: Trp1 \\
\hline byMM598 & byMM584 pMM729:: Leu2 pMM815:: Ura3 pMM779:: Trp1 \\
\hline byMM599 & byMM584 pMM729:: Leu2 pMM815:: Ura3 pMM790:: Trp1 \\
\hline byMM602 & byMM584 pMM729:: Leu2 pMM774:: Ura3 \\
\hline byMM603 & byMM584 pMM724:: Leu2 pMM814:: Ura3 \\
\hline byMM604 & byMM584 pMM729:: Leu2 pMM814:: Ura3 \\
\hline byMM622 & byMM584 pMM729:: Leu2 pMM774:: Ura3 pMM755:: Trp1 \\
\hline byMM623 & byMM584 pMM729:: Leu2 pMM774:: Ura3 pMM759:: Trp1 \\
\hline byMM624 & byMM584 pMM724:: Leu2 pMM815:: Ura3 pMM820:: Trp1 \\
\hline byMM625 & byMM584 pMM729:: Leu2 pMM776:: Ura3 pMM820:: Trp1 \\
\hline byMM626 & byMM584 pMM729:: Leu2 pMM776:: Ura3 pMM818:: Trp1 \\
\hline byMM636 & byMM584 pMM724:: Leu2 pMM814:: Ura3 pMM755:: Trp1 \\
\hline byMM637 & byMM584 pMM724:: Leu2 pMM814:: Ura3 pMM759:: Trp1 \\
\hline byMM653 & byMM584 pMM724:: Leu2 pMM815:: Ura3 pMM818:: Trp1 \\
\hline byMM654 & byMM584 pMM729:: Leu2 pMM815:: Ura3 pMM820:: Trp1 \\
\hline byMM655 & byMM584 pMM729:: Leu2 pMM815:: Ura3 pMM818:: Trp1 \\
\hline byMM656 & byMM584 pMM724:: Leu2 pMM814:: Ura3 pMM836:: Trp1 \\
\hline byMM657 & byMM584 pMM724:: Leu2 pMM814:: Ura3 pMM834:: Trp1 \\
\hline byMM658 & byMM584 pMM729:: Leu2 pMM814:: Ura3 pMM755:: Trp1 \\
\hline byMM659 & byMM584 pMM729:: Leu2 pMM814:: Ura3 pMM759:: Trp1 \\
\hline byMM660 & byMM584 pMM729:: Leu2 pMM814:: Ura3 pMM836:: Trp1 \\
\hline byMM661 & byMM584 pMM729:: Leu2 pMM814:: Ura3 pMM834:: Trp1 \\
\hline byMM662 & byMM584 pMM724:: Leu2 pMM774:: Ura3 pMM836:: Trp1 \\
\hline byMM663 & byMM584 pMM724:: Leu2 pMM774:: Ura3 pMM834:: Trp1 \\
\hline
\end{tabular}




\begin{tabular}{|c|c|}
\hline byMM664 & byMM584 pMM724:: Leu2 pMM776:: Ura3 pMM820:: Trp1 \\
\hline byMM665 & byMM584 pMM724:: Leu2 pMM776:: Ura3 pMM818:: Trp1 \\
\hline byMM680 & byMM584 pMM724:: Leu2 pMM846:: Ura3 \\
\hline byMM681 & byMM584 pMM724:: Leu2 pMM847:: Ura3 \\
\hline byMM682 & byMM584 pMM729:: Leu2 pMM846:: Ura3 \\
\hline byMM683 & byMM584 pMM729:: Leu2 pMM847:: Ura3 \\
\hline byMM684 & byMM584 pMM729:: Leu2 pMM848:: Ura3 \\
\hline byMM685 & byMM584 pMM724:: Leu2 pMM848:: Ura3 \\
\hline byMM686 & byMM584 pMM729:: Leu2 pMM849:: Ura3 \\
\hline byMM687 & byMM584 pMM724:: Leu2 pMM849:: Ura3 \\
\hline byMM712 & byMM584 pMM724:: Leu2 pMM846:: Ura3 pMM755:: Trp1 \\
\hline byMM713 & byMM584 pMM724:: Leu2 pMM846:: Ura3 pMM834:: Trp1 \\
\hline byMM714 & byMM584 pMM724:: Leu2 pMM847:: Ura3 pMM779:: Trp1 \\
\hline byMM715 & byMM584 pMM724:: Leu2 pMM847:: Ura3 pMM790:: Trp1 \\
\hline byMM716 & byMM584 pMM724:: Leu2 pMM847:: Ura3 pMM820:: Trp1 \\
\hline byMM717 & byMM584 pMM724:: Leu2 pMM847:: Ura3 pMM818:: Trp1 \\
\hline byMM718 & byMM584 pMM729:: Leu2 pMM846:: Ura3 pMM755:: Trp1 \\
\hline byMM719 & byMM584 pMM729:: Leu2 pMM846:: Ura3 pMM759:: Trp1 \\
\hline byMM720 & byMM584 pMM729:: Leu2 pMM846:: Ura3 pMM834:: Trp1 \\
\hline byMM721 & byMM584 pMM729:: Leu2 pMM846:: Ura3 pMM836:: Trp1 \\
\hline byMM722 & byMM584 pMM729:: Leu2 pMM847:: Ura3 pMM820:: Trp1 \\
\hline byMM723 & byMM584 pMM729:: Leu2 pMM847:: Ura3 pMM790:: Trp1 \\
\hline byMM724 & byMM584 pMM729:: Leu2 pMM847:: Ura3 pMM818:: Trp1 \\
\hline byMM738 & byMM584 pMM724:: Leu2 pMM846:: Ura3 pMM850:: Trp1 \\
\hline byMM739 & byMM584 pMM729:: Leu2 pMM846:: Ura3 pMM850:: Trp1 \\
\hline byMM740 & byMM584 pMM729:: Leu2 pMM847:: Ura3 pMM779:: Trp1 \\
\hline byMM742 & byMM584 pMM724:: Leu2 pMM848:: Ura3 pMM755:: Trp1 \\
\hline byMM743 & byMM584 pMM724:: Leu2 pMM848:: Ura3 pMM834:: Trp1 \\
\hline byMM744 & byMM584 pMM724:: Leu2 pMM849:: Ura3 pMM818:: Trp1 \\
\hline byMM745 & byMM584 pMM724:: Leu2 pMM849:: Ura3 pMM820:: Trp1 \\
\hline byMM746 & byMM584 pMM729:: Leu2 pMM849:: Ura3 pMM820:: Trp1 \\
\hline byMM747 & byMM584 pMM729:: Leu2 pMM848:: Ura3 pMM834:: Trp1 \\
\hline byMM748 & byMM584 pMM729:: Leu2 pMM849:: Ura3 pMM779:: Trp1 \\
\hline byMM749 & byMM584 pMM729:: Leu2 pMM849:: Ura3 pMM790:: Trp1 \\
\hline byMM750 & byMM584 pMM729:: Leu2 pMM848:: Ura3 pMM755:: Trp1 \\
\hline byMM755 & byMM584 pMM724:: Leu2 pMM848:: Ura3 pMM759:: Trp1 \\
\hline byMM756 & byMM584 pMM724:: Leu2 pMM849:: Ura3 pMM779:: Trp1 \\
\hline byMM757 & byMM584 pMM729:: Leu2 pMM849:: Ura3 pMM818:: Trp1 \\
\hline byMM758 & byMM584 pMM729:: Leu2 pMM848:: Ura3 pMM836:: Trp1 \\
\hline byMM795 & byMM584 pMM429:: Trp1 \\
\hline byMM796 & byMM584 pMM429:: Trp1 pMM879:: Ura3 \\
\hline byMM797 & byMM584 pMM429:: Trp1 pMM880:: Ura3 \\
\hline byMM799 & byMM584 pMM429:: Trp1 \\
\hline byMM800 & byMM584 pMM429:: Trp1 pMM784:: His3 \\
\hline
\end{tabular}




\begin{tabular}{|c|c|}
\hline byMM801 & byMM584 pMM429:: Trp1 pMM895:: His3 \\
\hline byMM802 & byMM584 pMM429:: Trp1 pMM522:: His3 \\
\hline byMM803 & byMM584 pMM429:: Trp1 pMM880:: Ura3 pMM882:: Leu2 \\
\hline byMM804 & byMM584 pMM429:: Trp1 pMM879:: Ura3 pMM882:: Leu2 \\
\hline byMM820 & byMM584 pMM429:: Trp1 pMM879:: Ura3 pMM882:: Leu2 pMM784:: His3 \\
\hline byMM821 & byMM584 pMM429:: Trp1 pMM879:: Ura3 pMM882:: Leu2 pMM522:: His3 \\
\hline byMM822 & byMM584 pMM429:: Trp1 pMM879:: Ura3 pMM882:: Leu2 pMM895:: His3 \\
\hline byMM824 & byMM584 pMM429:: Trp1 pMM895:: His3 pMM939:: Ura3 \\
\hline byMM825 & byMM584 pMM429:: Trp1 pMM522:: His3 pMM939:: Ura3 \\
\hline byMM826 & byMM584 pMM724:: Leu2 pMM914:: Ura3 \\
\hline byMM827 & byMM584 pMM724:: Leu2 pMM913:: Ura3 \\
\hline byMM828 & byMM584 pMM729:: Leu2 pMM914:: Ura3 \\
\hline byMM829 & byMM584 pMM729:: Leu2 pMM913:: Ura3 \\
\hline byMM830 & byMM584 pMM429:: Trp1 pMM915:: Ura3 \\
\hline byMM835 & byMM584 pMM429:: Trp1 pMM898:: Ura3 \\
\hline byMM836 & byMM584 pMM429:: Trp1 pMM939:: Ura3 \\
\hline byMM837 & byMM584 pMM729:: Leu2 pMM815:: Ura3 pMM875:: Trp1 \\
\hline byMM838 & byMM584 pMM429:: Trp1 pMM880:: Ura3 pMM938:: Leu \\
\hline byMM839 & byMM584 pMM729:: Leu2 pMM848:: Ura3 pMM759:: Trp1 \\
\hline byMM840 & byMM584 pMM724:: Leu2 pMM848:: Ura3 pMM836:: Trp1 \\
\hline byMM841 & byMM584 pMM724:: Leu2 pMM849:: Ura3 pMM790:: Trp1 \\
\hline byMM908 & byMM584 pMM729:: Leu2 pMM976:: Ura3 \\
\hline byMM909 & byMM584 pMM729:: Leu2 pMM1005:: Ura3 \\
\hline byMM910 & byMM584 pMM729:: Leu2 pMM848:: Ura3 pMM986:: Trp1 \\
\hline byMM911 & byMM584 pMM724:: Leu2 pMM848:: Ura3 pMM986:: Trp1 \\
\hline byMM912 & byMM584 pMM724:: Leu2 pMM774:: Ura3 pMM986:: Trp1 \\
\hline byMM913 & byMM584 pMM729:: Leu2 pMM774:: Ura3 pMM986:: Trp1 \\
\hline byMM914 & byMM584 pMM724:: Leu2 pMM976:: Ura3 \\
\hline byMM915 & byMM584 pMM724:: Leu2 pMM1005:: Ura3 \\
\hline byMM1029 & byMM584 pMM724:: Leu2 pMM849:: Ura3 pMM875:: Trp1 \\
\hline byMM1030 & byMM584 pMM729:: Leu2 pMM849:: Ura3 pMM875:: Trp1 \\
\hline byMM1031 & byMM584 pMM724:: Leu2 pMM776:: Ura3 pMM875:: Trp1 \\
\hline byMM1032 & byMM584 pMM729:: Leu2 pMM776:: Ura3 pMM875:: Trp1 \\
\hline byMM1033 & byMM584 pMM729:: Leu2 pMM774:: Ura3 pMM836:: Trp1 \\
\hline byMM1034 & byMM584 pMM729:: Leu2 pMM774:: Ura3 pMM834:: Trp1 \\
\hline byMM1035 & byMM584 pMM724:: Leu2 pMM848:: Ura3 pMM850:: Trp1 \\
\hline byMM1036 & byMM584 pMM729:: Leu2 pMM848:: Ura3 pMM850:: Trp1 \\
\hline byMM1037 & byMM584 pMM724:: Leu2 pMM774:: Ura3 pMM850:: Trp1 \\
\hline byMM1038 & byMM584 pMM729:: Leu2 pMM774:: Ura3 pMM850:: Trp1 \\
\hline byMM1039 & byMM584 pMM724:: Leu2 pMM914:: Ura3 pMM751:: Trp1 \\
\hline byMM1040 & byMM584 pMM724:: Leu2 pMM914:: Ura3 pMM761:: Trp1 \\
\hline byMM1041 & byMM584 pMM724:: Leu2 pMM914:: Ura3 pMM745:: Trp1 \\
\hline byMM1042 & byMM584 pMM729:: Leu2 pMM914:: Ura3 pMM751:: Trp1 \\
\hline byMM1043 & byMM584 pMM729:: Leu2 pMM914:: Ura3 pMM761:: Trp1 \\
\hline
\end{tabular}




\begin{tabular}{|c|c|}
\hline byMM1044 & byMM584 pMM729:: Leu2 pMM914:: Ura3 pMM745:: Trp1 \\
\hline byMM1045 & byMM584 pMM724:: Leu2 pMM916:: Ura3 \\
\hline byMM1046 & byMM584 pMM724:: Leu2 pMM916:: Ura3 pMM751:: Trp1 \\
\hline byMM1047 & byMM584 pMM724:: Leu2 pMM916:: Ura3 pMM761:: Trp1 \\
\hline byMM1048 & byMM584 pMM724:: Leu2 pMM916:: Ura3 pMM745:: Trp1 \\
\hline byMM1049 & byMM584 pMM729:: Leu2 pMM916:: Ura3 \\
\hline byMM1050 & byMM584 pMM729:: Leu2 pMM916:: Ura3 pMM751:: Trp1 \\
\hline byMM1051 & byMM584 pMM729:: Leu2 pMM916:: Ura3 pMM761:: Trp1 \\
\hline byMM1052 & byMM584 pMM729:: Leu2 pMM916:: Ura3 pMM745:: Trp1 \\
\hline byMM1053 & byMM584 pMM724:: Leu2 pMM847:: Ura3 pMM875:: Trp1 \\
\hline byMM1054 & byMM584 pMM729:: Leu2 pMM847:: Ura3 pMM875:: Trp1 \\
\hline byMM1055 & byMM584 pMM724:: Leu2 pMM815:: Ura3 pMM875:: Trp1 \\
\hline byMM1056 & byMM584 pMM724:: Leu2 pMM846:: Ura3 pMM759:: Trp1 \\
\hline byMM1057 & byMM584 pMM724:: Leu2 pMM846:: Ura3 pMM836:: Trp1 \\
\hline byMM1058 & byMM584 pMM724:: Leu2 pMM814:: Ura3 pMM850:: Trp1 \\
\hline byMM1059 & byMM584 pMM729:: Leu2 pMM814:: Ura3 pMM850:: Trp1 \\
\hline byMM1060 & byMM584 pMM724:: Leu2 pMM913:: Ura3 pMM751:: Trp1 \\
\hline byMM1061 & byMM584 pMM724:: Leu2 pMM913:: Ura3 pMM761:: Trp1 \\
\hline byMM1062 & byMM584 pMM724:: Leu2 pMM913:: Ura3 pMM745:: Trp1 \\
\hline byMM1063 & byMM584 pMM729:: Leu2 pMM913:: Ura3 pMM751:: Trp1 \\
\hline byMM1064 & byMM584 pMM729:: Leu2 pMM913:: Ura3 pMM761:: Trp1 \\
\hline byMM1065 & byMM584 pMM729:: Leu2 pMM913:: Ura3 pMM745:: Trp1 \\
\hline byMM1066 & byMM584 pMM724:: Leu2 pMM978:: Ura3 \\
\hline byMM1067 & byMM584 pMM724:: Leu2 pMM978:: Ura3 pMM751:: Trp1 \\
\hline byMM1068 & byMM584 pMM724:: Leu2 pMM978:: Ura3 pMM761:: Trp1 \\
\hline byMM1069 & byMM584 pMM724:: Leu2 pMM978:: Ura3 pMM745:: Trp1 \\
\hline byMM1070 & byMM584 pMM729:: Leu2 pMM978:: Ura3 \\
\hline byMM1071 & byMM584 pMM729:: Leu2 pMM978:: Ura3 pMM751:: Trp1 \\
\hline byMM1072 & byMM584 pMM729:: Leu2 pMM978:: Ura3 pMM761:: Trp1 \\
\hline byMM1073 & byMM584 pMM729:: Leu2 pMM978:: Ura3 pMM745:: Trp1 \\
\hline byMM1074 & byMM584 pMM429:: Trp1 pMM880:: Ura3 pMM938:: Leu pMM784:: His3 \\
\hline byMM1075 & byMM584 pMM429:: Trp1 pMM880:: Ura3 pMM938:: Leu pMM522:: His3 \\
\hline byMM1076 & byMM584 pMM429:: Trp1 pMM915:: Ura3 pMM940:: Trp1 \\
\hline byMM1077 & byMM584 pMM429:: Trp1 pMM879:: Ura3 pMM882:: Leu2 pMM941:: His3 \\
\hline byMM1078 & byMM584 pMM729:: Leu2 pMM847:: Ura3 pMM779:: Trp1 pMM784:: His3 \\
\hline byMM1079 & byMM584 pMM729:: Leu2 pMM847:: Ura3 pMM779:: Trp1 pMM522:: His3 \\
\hline byMM1080 & byMM584 pMM724:: Leu2 pMM815:: Ura3 pMM779:: Trp1 pMM784:: His3 \\
\hline byMM1081 & byMM584 pMM724:: Leu2 pMM815:: Ura3 pMM779:: Trp1 pMM522:: His3 \\
\hline byMM1082 & byMM584 pMM429:: Trp1 pMM880:: Ura3 pMM938:: Leu pMM1016:: His3 \\
\hline byMM1083 & $\begin{array}{l}\text { byMM584 pMM724:: Leu2 pMM815:: Ura3 pMM779:: Trp1 pMM1016:: } \\
\text { His3 }\end{array}$ \\
\hline byMM1084 & byMM584 pMM729:: Leu2 pMM846:: Ura3 pMM755:: Trp1 pMM784:: His3 \\
\hline byMM1085 & byMM584 pMM729:: Leu2 pMM846:: Ura3 pMM755:: Trp1 pMM895:: His3 \\
\hline byMM1086 & byMM584 pMM729:: Leu2 pMM846:: Ura3 pMM755:: Trp1 pMM941:: His3 \\
\hline
\end{tabular}




\begin{tabular}{|c|c|}
\hline byMM1087 & byMM584 pMM729:: Leu2 pMM846:: Ura3 pMM755:: Trp1 pMM522:: His3 \\
\hline byMM1088 & byMM584 pMM729:: Leu2 pMM846:: Ura3 pMM755:: Trp1 pMM917:: His3 \\
\hline byMM1089 & byMM584 pMM729:: Leu2 pMM846:: Ura3 pMM755:: Trp1 pMM977:: His3 \\
\hline byMM1090 & byMM584 pMM729:: Leu2 pMM846:: Ura3 pMM755:: Trp1 pMM975:: His3 \\
\hline byMM1091 & $\begin{array}{l}\text { byMM584 pMM729:: Leu2 pMM846:: Ura3 pMM755:: Trp1 pMM1015:: } \\
\text { His3 }\end{array}$ \\
\hline byMM1092 & byMM584 pMM724:: Leu2 pMM814:: Ura3 pMM834:: Trp1 pMM784:: His3 \\
\hline byMM1093 & byMM584 pMM724:: Leu2 pMM814:: Ura3 pMM834:: Trp1 pMM895:: His3 \\
\hline byMM1094 & byMM584 pMM724:: Leu2 pMM814:: Ura3 pMM834:: Trp1 pMM941:: His3 \\
\hline byMM1095 & byMM584 pMM724:: Leu2 pMM814:: Ura3 pMM834:: Trp1 pMM522:: His3 \\
\hline byMM1096 & byMM584 pMM724:: Leu2 pMM814:: Ura3 pMM834:: Trp1 pMM917:: His3 \\
\hline byMM1097 & byMM584 pMM724:: Leu2 pMM814:: Ura3 pMM834:: Trp1 pMM977:: His3 \\
\hline byMM1098 & byMM584 pMM724:: Leu2 pMM814:: Ura3 pMM834:: Trp1 pMM975:: His3 \\
\hline byMM1099 & $\begin{array}{l}\text { byMM584 pMM724:: Leu2 pMM814:: Ura3 pMM834:: Trp1 pMM1015:: } \\
\text { His3 }\end{array}$ \\
\hline byMM1100 & $\begin{array}{l}\text { byMM584 pMM724:: Leu2 pMM814:: Ura3 pMM834:: Trp1 pMM1016:: } \\
\text { His3 }\end{array}$ \\
\hline byMM1101 & $\begin{array}{l}\text { byMM584 pMM724:: Leu2 pMM814:: Ura3 pMM834:: Trp1 pMM1017:: } \\
\text { His3 }\end{array}$ \\
\hline byMM1102 & byMM584 pMM724:: Leu2 pMM814:: Ura3 pMM834:: Trp1 pMM987:: His3 \\
\hline byMM1103 & $\begin{array}{l}\text { byMM584 pMM724:: Leu2 pMM814:: Ura3 pMM834:: Trp1 pMM1018:: } \\
\text { His3 }\end{array}$ \\
\hline byMM1104 & $\begin{array}{l}\text { byMM584 pMM724:: Leu2 pMM814:: Ura3 pMM834:: Trp1 pMM1019:: } \\
\text { His3 }\end{array}$ \\
\hline byMM1105 & $\begin{array}{l}\text { byMM584 pMM724:: Leu2 pMM814:: Ura3 pMM834:: Trp1 pMM1020:: } \\
\text { His3 }\end{array}$ \\
\hline byMM1106 & $\begin{array}{l}\text { byMM584 pMM429:: Trp1 pMM879:: Ura3 pMM882:: Leu2 pMM1016:: } \\
\text { His3 }\end{array}$ \\
\hline byMM1107 & $\begin{array}{l}\text { byMM584 pMM429:: Trp1 pMM879:: Ura3 pMM882:: Leu2 pMM1017:: } \\
\text { His3 }\end{array}$ \\
\hline byMM1108 & byMM584 pMM429:: Trp1 pMM879:: Ura3 pMM882:: Leu2 pMM987:: His3 \\
\hline byMM1109 & $\begin{array}{l}\text { byMM584 pMM429:: Trp1 pMM879:: Ura3 pMM882:: Leu2 pMM1018:: } \\
\text { His3 }\end{array}$ \\
\hline byMM1110 & $\begin{array}{l}\text { byMM584 pMM429:: Trp1 pMM879:: Ura3 pMM882:: Leu2 pMM1019:: } \\
\text { His3 }\end{array}$ \\
\hline byMM1111 & $\begin{array}{l}\text { byMM584 pMM429:: Trp1 pMM879:: Ura3 pMM882:: Leu2 pMM1020:: } \\
\text { His3 }\end{array}$ \\
\hline byMM1112 & byMM584 pMM429:: Trp1 pMM939:: Ura3 pMM937:: His3 \\
\hline byMM1113 & byMM584 pMM429:: Trp1 pMM939:: Ura3 pMM932:: His3 \\
\hline byMM1114 & byMM584 pMM429:: Trp1 pMM939:: Ura3 pMM882:: Leu2 \\
\hline byMM1115 & byMM584 pMM429:: Trp1 pMM939:: Ura3 pMM937:: His3 pMM882:: Leu2 \\
\hline byMM1116 & byMM584 pMM429:: Trp1 pMM939:: Ura3 pMM932:: His3 pMM882:: Leu2 \\
\hline byMM1117 & byMM584 pMM429:: Trp1 pMM898:: Ura3 pMM937:: His3 \\
\hline byMM1118 & byMM584 pMM429:: Trp1 pMM898:: Ura3 pMM932:: His3 \\
\hline
\end{tabular}




\begin{tabular}{ll}
\hline byMM1119 & byMM584 pMM429:: Trp1 pMM898:: Ura3 pMM882:: Leu2 \\
byMM1120 & byMM584 pMM429:: Trp1 pMM898:: Ura3 pMM937:: His3 pMM882:: Leu2 \\
byMM1121 & byMM584 pMM429:: Trp1 pMM898:: Ura3 pMM932:: His3 pMM882:: Leu2 \\
byMM1122 & byMM584 pMM724:: Leu2 pMM774:: Ura3 pMM756:: Trp1 \\
byMM1123 & byMM584 pMM724:: Leu2 pMM774:: Ura3 pMM760:: Trp1 \\
byMM1124 & byMM584 pMM724:: Leu2 pMM774:: Ura3 pMM835:: Trp1 \\
byMM1125 & byMM584 pMM724:: Leu2 pMM774:: Ura3 pMM837:: Trp1 \\
byMM1126 & byMM584 pMM724:: Leu2 pMM814:: Ura3 pMM756:: Trp1 \\
byMM1127 & byMM584 pMM724:: Leu2 pMM814:: Ura3 pMM760:: Trp1 \\
byMM1128 & byMM584 pMM724:: Leu2 pMM814:: Ura3 pMM835:: Trp1 \\
byMM1129 & byMM584 pMM724:: Leu2 pMM814:: Ura3 pMM837:: Trp1 \\
\hline
\end{tabular}

Table S16. qPCR primers used in this work.

\begin{tabular}{ll}
\hline Name & Primer sequences \\
\hline ot398 & 5'-TGGTTTGGCTGGTGTTGATA-3' \\
ot399 & 5'-CGAAGTGAGAACGTTCAAA-3' \\
ot400 & 5'-CTTTATCGGAAGGACCGACA-3' \\
ot401 & 5'-TCAACAAAGCGTTCTCATGC-3' \\
ot279 & 5'-CAGGTATTGCCGAAAGAA-3' \\
ot280 & 5'-CCACATTTGTTGGAAGGTA-3' \\
ot441 & 5'-GTCGTATCCAGTGCAGGGTCCGAGGTATTCGCA \\
& CTGGATACGACTAACTG-3' \\
ot444 & 5'-GTCGTATCCAGTGCAGGGTCCGAGGTATTCGCA \\
& CtGGATACGACTGGGTG-3' \\
ot442 & 5'-GCGCGTAGATCCCTGGGTGTATATA-3' \\
ot443 & 5'-GCGCGCG GTAGATCATAACTGTATATA-3' \\
\hline
\end{tabular}

Table S17. DNA sequences used in this work.

\begin{tabular}{ll}
\hline DNA fragments & Sequences \\
\hline $\begin{array}{l}\text { lexOpR- } \\
\text { truncated- } \\
\text { pCYC1core }\end{array}$ & CAGGCAACTTTAGTGCTGACAtttaCATAACTGTATATACACCCAGG \\
& AAAACTCTTGTTTTCTTCTTTTCTCTAAATATTCTTTCCTTATACATt \\
& AGGACCTTTGCAGCATAAATTAC \\
\hline 3xlexOpR- & TGTAAAACGACGGCCAGTGAGCGCGCGTAATACGACTCACTATA \\
truncated- & GGGCGAATTGGGTACCCGAGCAGATCCGCCAGGCGTGTTACAT \\
pCYC1core & AACTGTATATACACCCAGGGCAAAAAAAAAAGCGTGGATGGCCT \\
& TTACATAACTGTATATACACCCAGGGCAAAAGGCAACTTTAGTGC \\
\hline
\end{tabular}


TGACATTTACATAACTGTATATACACCCAGGGCAAAGACACATGAT CATATGGCATGCATGTGCTCTGTATGTATATAAAACTCTTGTTTTC TTCTTTTCTCTAAATATTCTTTCCTTATACATTAGGACCTTTGCAGC ATAAATTAC

\begin{tabular}{|c|c|}
\hline \multirow[t]{13}{*}{ pGAL1 } & ATATACATATCCATATCTAATCTTACTTATATGTTGTGGAAATGTAA \\
\hline & AGAGCCCCATTATCTTAGCCTAAAAAAACCTTCTCTTTGGAACTTT \\
\hline & CAGTAATACGCTTAACTGCTCATTGCTATATTGAAGTACGGATTAG \\
\hline & AAGCCGCCGAGCGGGTGACAGCCCTCCGAAGGAAGACTCTCCTC \\
\hline & CGTGCGTCCTCGTCTTCACCGGTCGCGTTCCTGAAACGCAGATG \\
\hline & TGCCTCGCGCCGCACTGCTCCGAACAATAAAGATTCTACAATACT \\
\hline & AGCTTTTATGGTTATGAAGAGGAAAAATTGGCAGTAACCTGGCCC \\
\hline & CACAAACCTTCAAATGAACGAATCAAATTAACAACCATAGGATGAT \\
\hline & AATGCGATTAGTTTTTTAAGCCTTATTTCTGGGGTAATTAATCAGCG \\
\hline & AAGCGATGATTTTTGATCTATTAACAGATATATAAATGCAAAAACT \\
\hline & GCATAACCACTTTAACTAATACTTTCAACATTTTCGGTTTGTATTAC \\
\hline & TTCTTATTCAAATGTAATAAAAGTATCAACAAAAAATTGTTAATATA \\
\hline & ССТСТАTACTTTAACGTCAAGGAGAAAAAACtata \\
\hline \multirow[t]{11}{*}{ pGPD } & cagttcgagtttatcattatcaatactgccatttcaaagaatacgtaaataattaatagtagtgatttc \\
\hline & ctaactttatttagtcaaaaaattagccttttaattctgctgtaacccgtacatgcccaaaatagggg \\
\hline & gcgggttacacagaatatataacatcgtaggtgtctgggtgaacagtttattcctggcatccactaa \\
\hline & atataatggagcccgcttttaagctggcatccagaaaaaaaaagaatcccagcaccaaaatat \\
\hline & tgtttcttcaccaaccatcagttcataggtccattctcttagcgcaactacagagaacaggggcac \\
\hline & aaacaggcaaaaaacgggcacaacctcaatggagtgatgcaacctgcctggagtaaatgatg \\
\hline & acacaaggcaattgacccacgcatgtatctatctcattttcttacaccttctattaccttctgctctctct \\
\hline & gatttggaaaaagctgaaaaaaaaggttgaaaccagttccctgaaattattcccctacttgactaa \\
\hline & taagtatataaagacggtaggtattgattgtaattctgtaaatctatttcttaaacttcttaaattctacttt \\
\hline & tatagttagtctttttttagttttaaaacaccaagaacttagtttcgaataaacacacataaacaaac \\
\hline & ( \\
\hline \multirow[t]{13}{*}{ pADH1 } & GGGTGTACAATATGGACTTCCTCTTTTCTGGCAACCAAACCCATA \\
\hline & CATCGGGATTCCTATAATACCTTCGTTGGTCTCCCTAACATGTAG \\
\hline & GTGGCGGAGGGGAGATATACAATAGAACAGATACCAGACAAGAC \\
\hline & ATAATGGGCTAAACAAGACTACACCAATTACACTGCCTCATTGAT \\
\hline & GGTGGTACATAACGAACTAATACTGTAGCCCTAGACTTGATAGCC \\
\hline & АTCATCATATCGAAGTTTCACTACCСTTTTTCCATTTGCСАTCTATT \\
\hline & GAAGTAATAATAGGCGCATGCAACTTCTTTTCTTTTTTTTTTCTTTTC \\
\hline & TCTCTCCCCCGTTGTTGTCTCACCATATCCGCAATGACAAAAAAAT \\
\hline & GATGGAAGACACTAAAGGAAAAAATTAACGACAAAGACAGCACCA \\
\hline & ACAGATGTCGTTGTTCCAGAGCTGATGAGGGGTATCTCGAAGCA \\
\hline & CACGAAACTTTTTTCCTTCCTTCATTCACGCACACTACTCTCTAATG \\
\hline & AGCAACGGTATACGGCCTTCCTTCCAGTTACTTGAATTTGAAATAA \\
\hline & AAAAAAGTTTGCTGTCTTGCTATCAAGTATAAATAGACCTGCAATT \\
\hline
\end{tabular}




\begin{tabular}{|c|c|}
\hline & $\begin{array}{l}\text { ATTAATCTTTTGTTTCCTCGTCATTGTTCTCGTTCCСTTTCTTCCTT } \\
\text { GTTTCTTTTTCTGCACAATATTTCAAGCTATACCAAGCATACAATCA } \\
\text { АСTATCTCATATACA }\end{array}$ \\
\hline \multirow[t]{15}{*}{ pTEF2 } & GCTACCTATATTCCACCATAACATCAATCATGCGGTTGCTGGTGTA \\
\hline & TTTACCAATAATGTTTAATGTATATATATATATATATATGGGGCCGT \\
\hline & ATACTTACATATAGTAGATGTCAAGCGTAGGCGCTTCCCCTGCCG \\
\hline & GCTGTGAGGGCGCCATAACCAAGGTATCTATAGACCGCCAATCA \\
\hline & GCAAACTACCTCCGTACATTCATGTTGCACCCACACATTTATACAC \\
\hline & CCAGACCGCGACAAATTACCCATAAGGTTGTTTGTGACGGCGTC \\
\hline & GTACAAGAGAACGTGGGAACTTTTTAGGCTCACCAAAAAAGAAAG \\
\hline & AAAAAATACGAGTTGCTGACAGAAGCCTCAAGAAAAAAAAAATTCT \\
\hline & TCTTCGACTATGCTGGAGGCAGAGATGATCGAGCCGGTAGTTAA \\
\hline & CTATATATAGCTAAATTGGTTCCATCACCTTCTTTTCTGGTGTCGC \\
\hline & TCCTTCTAGTGCTATTTCTGGCTTTTCCTATTTTTTTTTTTTCCATTTT \\
\hline & ТСТTТСТСТСТTТСТААТАТАТАААТTСТСТTGСАТTTTСТАТTTTTС \\
\hline & TCTCTATCTATTCTACTTGTTTATTCCCTTCAAGGTTTTTTTTTAAA \\
\hline & GAGTACTTGTTTTTAGAATATACGGTCAACGAACTATAATTAACTA \\
\hline & AAC \\
\hline genCYC1t- & ACAGGCCCCTTTTCCTTTGTCGATATCATGTAATTAGTTATGTCAC \\
\hline \multirow[t]{6}{*}{ pCYC1noTATA } & GCTTACATTCACGCССTССTCCCACATCCGCTCTAACCGAAAAGG \\
\hline & AAGGAGTTAGACAACCTGAAGTCTAGGTCCCTATTTATTTTTTTTA \\
\hline & ATAGTTATGTTAGTATTAAGAACGTTATTTATATTTCAAATTTTTCTT \\
\hline & TTTTTTCTGTACAAACGCGTGTACGCATGTAACATTATATTCTTTCC \\
\hline & TTATACATtAGGACCTTTGCAGCATAAATTACTATACTTCTATAGAC \\
\hline & ACACAAACACAAATACACACACTAAATTAATA \\
\hline \multirow[t]{9}{*}{ pTEF1 } & ATAGCTTCAAAATGTTTCTACTCСTTTTTTTACTCTTCCAGATTTTCT \\
\hline & CGGACTCCGCGCATCGCCGTACCACTTCAAAACACCCAAGCACA \\
\hline & GCATACTAAATTTCCССTCTTTCTTCCTCTAGGGTGTCGTTAATTA \\
\hline & CCCGTACTAAAGGTTTGGAAAAGAAAAAAGAGACCGCCTCGTTTC \\
\hline & TTTTTCTTCGTCGAAAAAGGCAATAAAAATTTTTATCACGTTTCTTT \\
\hline & TTCTTGAAAATTTTTTTTTTTGATTTTTTTTCTCTTTCGATGACCTCCCA \\
\hline & TTGATATTTAAGTTAATAAACGGTCTTCAATTTCTCAAGTTTCAGTT \\
\hline & TCATTTTTCTTGTTCTATTACAACTTTTTTTTACTTCTTGCTCATTAGA \\
\hline & AAGAAAGCATAGCAATCTAATCTAAGTTT \\
\hline \multirow[t]{5}{*}{ pSNR52 } & ctttgaaaagataatgtatgattatgctttcactcatatttatacagaaacttgatgttttctttcgagTA \\
\hline & TATAcaaggtgattacatgtacgtttgaagtacaactctAgattttgtagtgccctcTTGGGC \\
\hline & TAGCGGTAAAGGtgcgcaTTTTTTcacaccctacaatgttctgttcaaaagattttggtc \\
\hline & aaacgctgtagaagtgaaagttggtgcgcatgtttcggCGTTCGAAACttctccgcagtga \\
\hline & aagataaatgatc \\
\hline \multirow[t]{3}{*}{ yEGFP } & ATGTCTAAAGGTGAAGAATTATTCACTGGTGTTGTCCCAATTTTGG \\
\hline & TTGAATTAGATGGTGATGTTAATGGTCACAAATTTTCTGTCTCCGG \\
\hline & TGAAGGTGAAGGTGATGCTACTTACGGTAAATTGACCTTAAAATTT \\
\hline
\end{tabular}




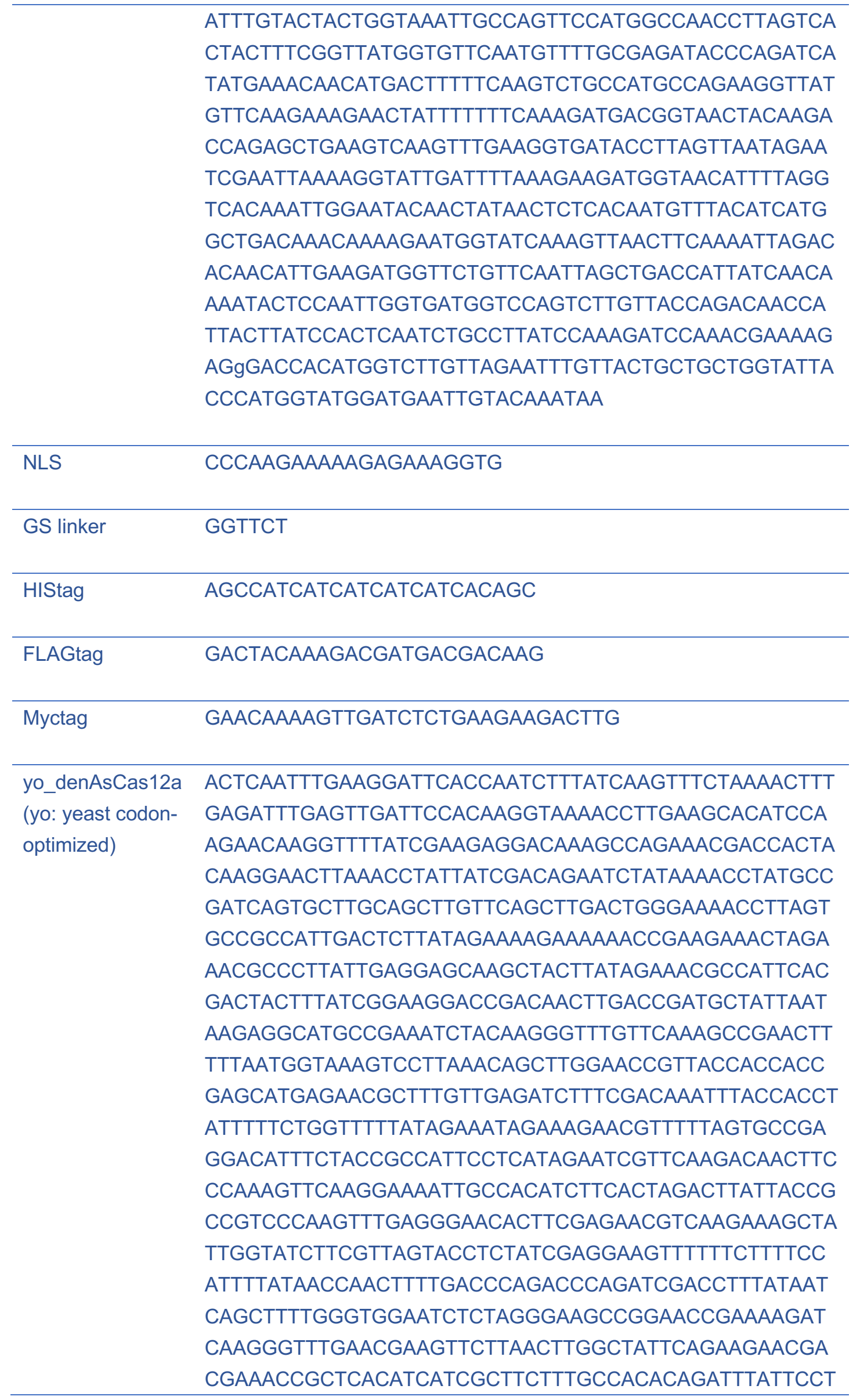


TTGTTTAAACAAATCTTGTCTGATAGGAACACTCTTTCTTTTATTCT TGAAGAATTTAAGTCTGACGAAGAAGTTATTCAAAGTTTCTGCAAG TACAAGACCCTTTTGAGGAATGAGAATGTCTTGGAGACTGCCGAG GCTTTGTTCAATGAGCTTAATTCTATTGACTTGACTCACATTTTTAT TTCTCATAAGAAATTGGAAACCATCAGTAGTGCCCTTTGCGACCA CTGGGACACCTTGAGGAACGCTCTTTATGAAAGAAGAATTTCTGA GTTGACCGGAAAGATCACTAAAAGTGCTAAGGAAAAAGTTCAAAG ATCTTTGAAACATGAAGATATTAACTTGCAAGAAATCATCTCTGCC GCCGGAAAGGAACTTTCTGAAGCTTTTAAGCAAAAGACCTCTGAG ATCCTTTCTCATGCCCATGCTGCTCTTGACCAGCCTCTTCCTACCA CCCTTAAGAAGCAAGAGGAGAAGGAGATCTTGAAAAGTCAATTGG ATAGTTTGCTTGGATTGTATCATTTGCTTGATTGGTTTGCCGTCGA CGAGAGTAACGAGGTCGACCCAGAGTTCAGTGCTAGACTTACTG GTATTAAATTGGAGATGGAACCAAGTTTGTCTTTTTACAATAAAGC TAGAAACTACGCTACTAAGAAACCTTACAGTGTCGAAAAGTTTAAG CTTAATTTCCAAATGCCTACTTTGGCTAGGGGATGGGACGTCAAT AGAGAGAAGAATAACGGTGCCATCCTTTTCGTCAAGAATGGACTT TATTACTTGGGAATCATGCCTAAACAAAAAGGTAGGTATAAAGCC CTTAGTTTCGAGCCAACCGAAAAAACCTCTGAGGGATTTGACAAA ATGTATTATGACTATTTTCCAGATGCCGCCAAGATGATCCCAAAAT GCAGTACTCAGTTGAAGGCTGTCACCGCCCACTTTCAGACTCACA CCACTCCAATCTTGCTTAGTAACAACTTCATTGAACCTCTTGAGAT TACTAAGGAAATCTATGATTTGAACAATCCAGAAAAAGAACCAAAA AAGTTCCAGACCGCCTACGCTAAGAAAACTGGCGATCAAAAGGG TTATAGAGAGGCTTTGTGCAAGTGGATCGATTTCACTAGAGATTTT CTTAGTAAGTATACTAAAACTACTTCTATCGACTTGTCTAGTCTTA GACCATCTAGTCAGTATAAGGATCTTGGAGAATATTACGCTGAAC TTAACCCTCTTTTGTATCATATTTCTTTTCAAAGAATCGCTGAAAAA GAGATCATGGATGCCGTTGAAACCGGTAAATTGTACTTGTTCCAA ATTTATAACAAGGACTTCGCCAAGGGTCATCACGGAAAGCCTAAT CTTCATACCCTTTACTGGACCGGACTTTTCTCTCCAGAGAACTTG GCTAAAACTAGTATTAAGTTGAATGGTCAAGCTGAATTGTTCTACA GACCTAAATCTAGAATGAAGAGGATGGCCCATAGATTGGGAGAG AAAATGCTTAATAAAAAGTTGAAGGACCAAAAGACCCCTATCCCA GATACCCTTTACCAAGAGCTTTATGATTATGTTAATCATAGGCTTA GTCATGATCTTAGTGACGAAGCTAGAGCCCTTTTGCCAAACGTCA TCACCAAGGAGGTCAGTCACGAGATCATCAAAGATAGAAGATTTA CCAGTGACAAATTCTTCTTTCATGTCCCTATTACCTTGAATTACCA AGCTGCCAATTCTCCAAGTAAGTTCAACCAGAGGGTCAACGCTTA CTTGAAGGAGCATCCAGAAACCCCTATTATCGGAATTGCTAGAGG TGAGAGAAACCTTATTTATATCACCGTCATCGACAGTACTGGTAA GATCCTTGAGCAGAGATCTTTGAATACCATTCAGCAGTTCGATTAT CAAAAGAAACTTGACAATAGAGAGAAAGAAAGGGTTGCCGCCAG ACAAGCTTGGTCTGTCGTCGGAACTATCAAAGACCTTAAGCAAGG 
TTATTTGAGTCAAGTTATTCATGAAATTGTTGATTTGATGATCCACT ACCAAGCCGTCGTTGTCCTTGAAAACTTGAATTTTGGATTTAAATC TAAAAGAACTGGAATCGCCGAGAAGGCCGTTTACCAACAGTTCGA GAAAATGTTGATCGATAAATTGAATTGTTTGGTCCTTAAAGACTAT CCAGCCGAGAAAGTCGGTGGTGTCCTTAACCCATACCAACTTACC GACCAGTTCACCTCTTTCGCCAAAATGGGAACCCAGTCTGGTTTC TTGTTCTATGTCCCAGCTCCATACACCTCTAAGATCGACCCACTTA CCGGTTTCGTCGACCCTTTTGTTTGGAAAACCATCAAAAATCACG AGAGTAGAAAACATTTCCTTGAAGGATTCGATTTCTTGCATTATGA CGTCAAAACCGGAGACTTTATTTTGCACTTTAAGATGAATAGAAAC TTGTCTTTCCAGAGGGGTTTGCCCGGTTTCATGCCAGCTTGGGAC ATTGTCTTTGAAAAGAATGAGACTCAGTTTGATGCCAAGGGAACC CCATTCATCGCTGGTAAGAGGATCGTCCCAGTTATCGAGAACCAT AGGTTCACTGGTAGGTATAGGGACCTTTACCCAGCTAACGAGCTT ATCGCCTTGCTTGAGGAGAAGGGAATCGTCTTTAGGGATGGTTCT AACATTCTTCCTAAATTGTTGGAGAACGACGACTCTCACGCTATC GATACCATGGTTGCTTTGATCAGATCTGTCTTGCAGATGAGGAAT TCTAATGCCGCCACCGGTGAGGACTATATTAACAGTCCAGTCAGA GACTTGAACGGTGTCTGCTTCGACAGTAGGTTCCAGAACCCAGA GTGGCCAATGGATGCCGATGCCAACGGAGCTTATCACATCGCCC TTAAGGGACAATTGTTGTTGAATCACCTTAAGGAAAGTAAAGATCT TAAGCTTCAGAATGGAATCAGTAACCAAGATTGGCTTGCCTATAT CCAAGAATTGAGAAAT

yo_dLbCas12a TCAAAGTTGGAAAAATTTACTAACTGTTATTCTTTGTCAAAGACTTT AAGATTTAAAGCTATACCTGTTGGTAAGACTCAAGAGAATATAGAT AATAAAAGATTGTTGGTCGAAGACGAAAAGAGGGCTGAAGATTAT AAAGGTGTTAAAAAGTTGTTGGATAGGTATTATTTGTCTTTCATTAA CGATGTCTTGCATTCTATTAAATTGAAAAACTTGAATAATTATATTT CTTTATTTCGTAAGAAAACTAGAACTGAAAAAGAAAATAAAGAGTT AGAAAACTTGGAAATAAATTTAAGAAAAGAAATAGCAAAGGCTTTC AAAGGTAATGAAGGTTATAAGTCATTATTTAAGAAGGATATTATAG AAACAATTTTGCCAGAATTCTTGGATGATAAGGATGAAATTGCTTT GGTCAATTCTTTCAACGGTTTTACTACAGCATTCACTGGCTTTTTT GACAATAGGGAAAACATGTTTTCTGAGGAAGCTAAGTCTACTTCT ATAGCTTTCAGATGTATTAATGAAAACTTGACTAGATATATTTCTAA TATGGATATATTTGAGAAGGTTGATGCTATTTTCGATAAGCATGAA GTTCAGGAGATTAAAGAGAAGATTTTAAATTCTGACTACGATGTTG AAGACTTTTTTGAGGGTGAATTTTTCAATTTTGTTTTGACTCAAGAA GGTATTGATGTTTATAATGCTATAATAGGAGGATTCGTCACAGAGT CTGGTGAGAAGATAAAGGGTTTGAATGAATACATTAATTTGTATAA CCAAAAGACAAAACAGAAATTGCCTAAATTTAAGCCATTGTACAAG CAAGTTTTATCAGATAGAGAATCATTGTCATTCTATGGTGAAGGTT ACACTTCTGATGAGGAAGTTTTGGAGGTCTTCAGAAATACATTGA 
ACAAAAACTCTGAAATATTTTCTTCTATTAAAAAATTGGAGAAATTA TTCAAAAATTTTGATGAGTATTCTTCAGCAGGTATTTTCGTCAAAAA TGGTCCTGCAATTTCAACTATTTCTAAAGATATTTTTGGTGAATGG AACGTTATTAGGGACAAATGGAACGCTGAATATGACGATATACAT TTGAAAAAGAAGGCTGTCGTCACAGAAAAGTATGAGGATGATAGA AGAAAGTCTTTTAAGAAAATTGGTTCATTCTCATTGGAACAATTGC AGGAGTACGCTGACGCTGACTTGTCAGTCGTTGAAAAGTTGAAG GAAATTATTATACAAAAAGTTGATGAAATTTACAAAGTCTACGGTT CTTCTGAGAAGTTATTTGATGCTGACTTTGTCTTAGAGAAATCTTT AAAGAAGAATGATGCTGTTGTTGCTATTATGAAGGACTTATTGGAT TCAGTTAAATCTTTCGAGAATTATATTAAAGCTTTTTTCGGTGAAG GAAAGGAGACTAACAGAGACGAATCTTTCTATGGTGATTTTGTTTT GGCTTATGATATATTATTGAAAGTTGACCACATTTACGATGCTATT AGAAACTACGTTACACAGAAGCCATACTCTAAGGACAAGTTCAAA TTGTACTTTCAAAACCCTCAGTTCATGGGTGGTTGGGATAAAGAC AAAGAAACAGATTATAGAGCTACTATTTTGAGATACGGTTCAAAAT ACTACTTAGCTATTATGGATAAGAAATATGCAAAATGCTTGCAGAA AATAGATAAAGACGATGTTAATGGTAATTATGAAAAAATTAATTATA AATTATTACCAGGACCAAACAAGATGTTACCAAAAGTTTTCTTTTC TAAAAAGTGGATGGCTTACTATAACCCTTCTGAAGACATACAGAAA ATTTACAAGAATGGTACATTTAAAAAGGGTGATATGTTTAATTTAAA TGACTGCCATAAGTTGATAGACTTCTTCAAAGATTCAATATCAAGG TATCCAAAATGGTCTAATGCATATGACTTTAATTTTTCAGAGACTG AGAAATATAAGGACATTGCTGGTTTCTATAGAGAAGTCGAAGAAC AAGGTTACAAGGTTTCTTTTGAGTCTGCTTCAAAGAAAGAAGTTGA TAAGTTGGTTGAAGAAGGTAAATTGTACATGTTTCAAATTTACAAC AAGGATTTTTCAGACAAATCTCATGGTACACCTAATTTGCACACAA TGTATTTCAAATTGTTGTTTGATGAAAACAACCACGGTCAGATAAG GTTGTCTGGTGGAGCTGAATTGTTTATGAGAAGAGCATCTTTGAA AAAGGAGGAGTTGGTCGTCCATCCTGCAAACTCTCCAATTGCTAA CAAGAACCCAGATAATCCAAAAAAGACTACTACATTGTCTTATGAC GTTTACAAGGATAAGAGATTCTCTGAGGATCAATATGAATTACATA TTCCAATTGCTATTAACAAGTGTCCAAAAAATATTTTCAAAATTAAT ACAGAGGTCAGAGTTTTATTAAAACACGATGATAACCCTTATGTCA TTGGTATAGCAAGGGGTGAAAGGAACTTGTTATATATTGTTGTCGT CGATGGTAAAGGTAATATTGTCGAACAATACTCTTTAAATGAGATT ATAAACAACTTTAATGGAATTAGAATTAAGACAGATTATCATTCATT GTTAGATAAGAAAGAGAAGGAAAGATTTGAAGCTAGACAAAATTG GACATCTATTGAAAATATTAAAGAATTGAAGGCTGGATACATTTCA CAAGTTGTCCATAAAATATGTGAATTAGTTGAAAAATACGATGCTG TTATTGCTTTGGCAGATTTGAACTCTGGTTTTAAGAATTCTAGGGT TAAAGTTGAGAAACAGGTTTATCAAAAGTTTGAAAAAATGTTAATA GATAAGTTGAACTATATGGTTGACAAAAAATCTAACCCTTGCGCTA CAGGTGGAGCTTTGAAAGGTTATCAAATAACTAACAAGTTCGAAT 
CATTTAAGTCAATGTCTACTCAAAATGGTTTCATTTTCTATATTCCT GCTTGGTTAACATCAAAGATTGACCCATCAACTGGTTTTGTCAATT TGTTGAAAACTAAGTACACTTCTATAGCAGATTCAAAAAAGTTCAT TTCATCTTTCGATAGAATTATGTATGTTCCTGAGGAGGATTTATTT GAGTTTGCTTTGGATTACAAAAATTTTTCTAGAACAGATGCTGATT ACATAAAGAAGTGGAAATTGTACTCTTACGGTAATAGAATTAGGAT TTTCAGAAACCCTAAGAAAAATAATGTTTTCGACTGGGAAGAAGTT TGCTTGACATCTGCTTATAAAGAATTGTTTAATAAATATGGAATAAA CTATCAACAAGGTGATATTAGAGCTTTATTGTGTGAACAGTCTGAC AAGGCTTTTTATTCTTCATTTATGGCTTTGATGTCATTAATGTTGCA GATGAGAAATTCTATTACAGGTAGAACTGACGTCGATTTCTTGATA TCTCCAGTTAAAAATTCTGATGGAATTTTCTATGATTCAAGAAACT ATGAAGCTCAAGAGAATGCTATTTTACCTAAAAATGCTGATGCTAA CGGAGCATATAATATTGCAAGGAAAGTCTTATGGGCTATTGGTCA ATTTAAGAAAGCTGAAGACGAAAAATTAGACAAAGTTAAAATAGCT ATTTCTAATAAAGAATGGTTAGAATACGCTCAAACTTCTGTTAAAC AC

yo_dMbCas12a TTGTTCCAAGATTTCACTCACTTGTACCCATTGTCTAAGACCGTTA GATTTGAGTTGAAGCCAATTGGTAGAACCTTGGAACATATTCACG CCAAGAATTTCTTGTCTCAAGACGAAACTATGGCCGACATGTACC AAAAGGTTAAGGTTATCTTGGATGACTACCACAGAGATTTCATTGC TGATATGATGGGTGAAGTCAAGTTGACTAAGTTGGCTGAATTCTA CGATGTCTACCTGAAGTTTAGAAAGAACCCAAAGGATGACGAATT GCAGAAACAATTGAAAGACTTGCAAGCCGTCTTGAGGAAAGAATC TGTTAAGCCTATTGGTAACGGTGGTAAGTACAAAGCTGGTCATGA TAGATTATTCGGTGCCAAGTTGTTCAAGGACGGTAAAGAATTGGG TGATTTGGCCAAGTTTGTTATTGCCCAAGAAGGTAAATCTTCTCCA AAATTGGCTCATTTGGCCCACTTTGAAAAGTTCTCTACTTACTTCA CTGGTTTCCACGACAACAGAAAGAACATGTATTCTGACGAAGATA AGCACACCGCTATTGCCTATAGATTGATTCACGAAAACTTGCCAA GATTCATCGACAACCTGCAAATTTTGACCACCATTAAGCAAAAACA CTCCGCCTTGTACGATCAGATTATTAACGAATTGACTGCCTCCGG TTTGGATGTTTCTTTGGCTTCTCATTTGGATGGTTACCATAAGTTG TTGACCCAAGAGGGTATTACTGCCTACAACAGAATTATCGGTGAA GTTAACGGTTACACCAACAAGCACAATCAAATCTGCCATAAGTCC GAAAGAATTGCTAAACTAAGACCATTGCACAAGCAGATCTTGTCT GATGGTATGGGTGTTTCTTTCCTGCCATCTAAATTTGCCGATGATT CTGAAATGTGCCAAGCTGTCAATGAGTTCTATAGACATTACGCTG ATGTTTTCGCCAAGGTCCAATCTTTGTTTGATGGTTTTGATGACCA CCAAAAGGATGGTATCTACGTTGAACATAAGAACCTGAACGAGTT GTCTAAGCAAGCTTTTGGTGATTTTGCTTTGTTGGGTAGAGTTTTG GACGGTTACTATGTTGATGTTGTCAACCCAGAATTCAACGAAAGA TTCGCTAAGGCTAAGACTGATAACGCTAAAGCTAAGTTGACCAAA 
GAGAAGGACAAGTTCATTAAGGGTGTTCATTCTTTGGCCTCATTA

GAACAAGCTATTAAGCACCATACTGCCAGACATGATGATGAATCA GTTCAAGCTGGTAAGTTGGGTCAATACTTCAAACATGGTTTGGCT GGTGTTGATAACCCAATCCAAAAGATCCATAACAACCACTCCACT ATCAAGGGTTTCTTGGAAAGGGAAAGACCTGCTGGTGAAAGAGC TTTGCCAAAGATTAAGTCTGGTAAGAACCCAGAAATGACCCAATT GAGACAGCTGAAAGAATTATTGGATAACGCTTTGAACGTTGCTCA CTTCGCTAAGTTGTTAATGACTAAGACTACCTTGGATAACCAGGAT GGTAATTTCTACGGTGAATTTGGTGTGTTGTATGATGAGTTGGCTA AGATTCCAACCTTGTACAACAAGGTTAGAGACTACTTGTCCCAAA AGCCATTCTCTACTGAAAAGTACAAGTTGAACTTCGGTAACCCTA CTTTGTTGAATGGTTGGGACTTGAACAAAGAAAAGGATAACTTCG GTGTCATCTTGCAAAAAGATGGTTGTTACTACTTGGCCTTGTTGGA TAAGGCTCACAAAAAGGTTTTTGATAACGCCCCAAACACCGGTAA GAATGTTTACCAAAAAATGATCTACAAGCTGCTGCCAGGTCCAAA CAAAATGTTGCCAAGAGTTTTCTTTGCCAAGTCGAACTTGGATTAC TACAATCCATCTGCTGAGTTGTTGGACAAATATGCTCAAGGTACTC ATAAGAAGGGTGACAACTTTAACTTGAAGGATTGCCATGCCTTGA TCGATTTCTTTAAGGCCGGTATTAACAAACACCCAGAATGGCAAA ATTTCGGCTTTAAGTTTTCCCCAACCTCCTCATATAGAGACTTGTC CGATTTTTACAGAGAAGTCGAACCACAAGGTTACCAAGTTAAGTT CGTTGATATTAACGCCGACTACATCGATGAATTGGTTGAACAAGG TCAGTTGTACTTGTTCCAGATCTACAACAAAGACTTCTCACCTAAA GCTCATGGTAAGCCAAACTTGCATACCTTGTATTTCAGAGCCTTGT TCTCCGAAGATAATTTGGCTAATCCAATCTACAAATTGAACGGTGA AGCCCAGATTTTCTACAGAAAAGCTTCTTTGGGTATGAACGAAAC CACCATTCATAGAGCCGGTGAAATTCTGGAAAACAAGAATCCAGA TAACCCCAAAGAAAGGGTTTTCACCTACGATATTATCAAGGACAG AAGATACACCCAGGATAAGTTCATGTTGCATGTTCCAATTACCATG AATTTCGGTGTTCAAGGTATGACCATCAAAGAGTTCAACAAGAAG GTTAACCAGTCCATCAGGCAATACGATGATGTTAACGTTATTGGT ATTGCCAGAGGTGAAAGGCACTTGTTGTATTTGACTGTCATTAACT CCAAGGGTGAGATCCTAGAACAAAGGTCTTTGAACGATATTACTA CCGCTTCTGCTAACGGTACTCAAATGACTACTCCATACCATAAGA TCTTGGACAAGAGAGAAATCGAAAGATTGAACGCTAGAGTTGGTT GGGGTGAAATTGAAACGATCAAAGAATTGAAGTCCGGCTACTTGT CTCATGTTGTTCATCAAGTTTCCCAGCTGATGTTGAAGTACAATGC TATAGTTGTTTTGGCCGACCTGAACTTTGGTTTTAAAAGAGGTAGA TTCAAGGTCGAGAAGCAAATCTACCAGAACTTCGAAAATGCCCTG ATCAAGAAGTTGAACCATTTGGAGTTGAAAGATAAGGCCGATGAC GAAATTGGTTCTTACAAAAATGCCTTGCAACTGACCAACAACTTCA CTGATTTGAAGAACATTGGTAAGCAGACCGGTTTCTTGTTCTATGT TCCAGCTTGGAATACCTCCAAGATTGATCCAGAAACTGGTTTCGT AGACTTGTTGAAACCTAGGTACGAAAACATTGCTCAATCCCAAGC 


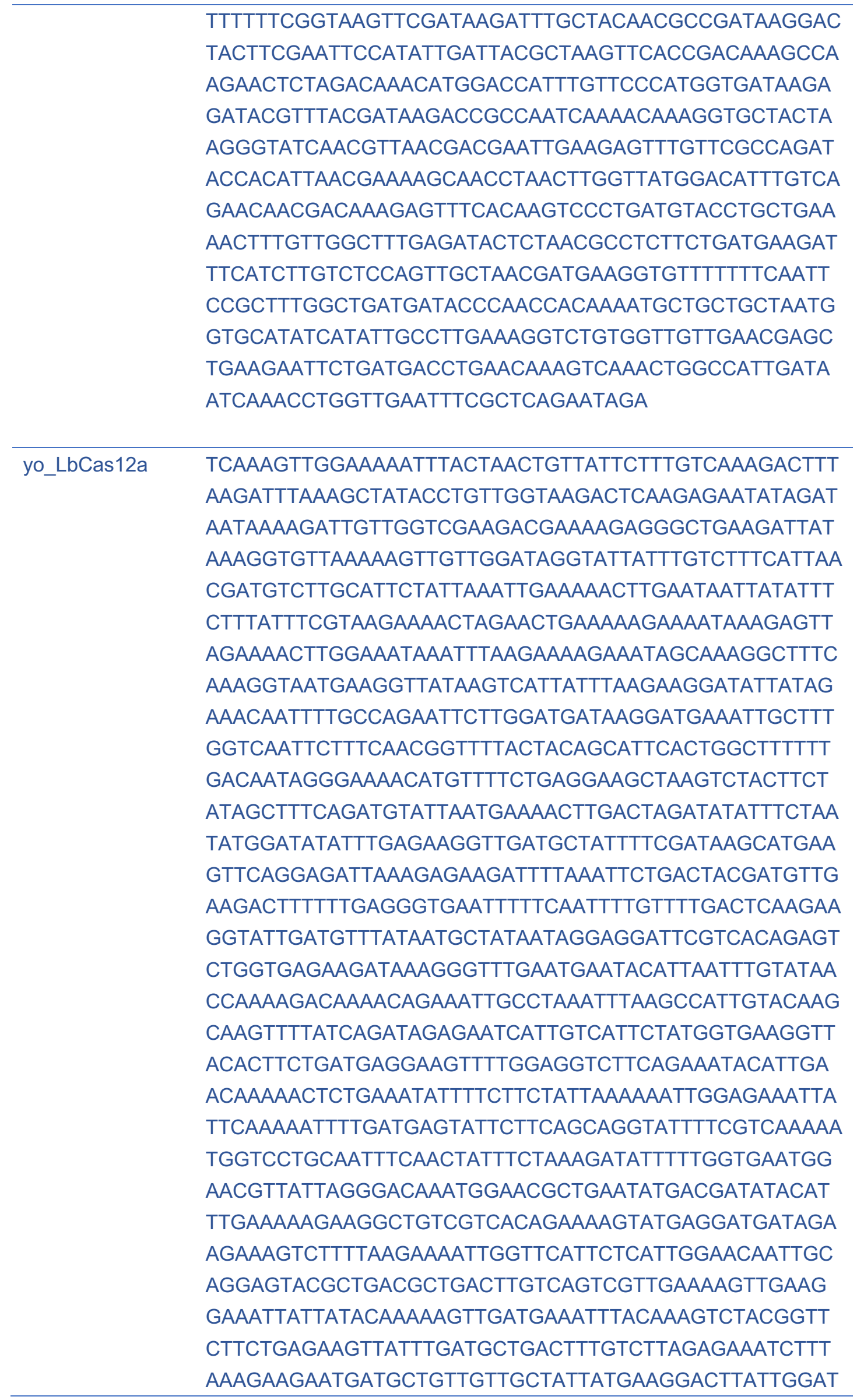


TCAGTTAAATCTTTCGAGAATTATATTAAAGCTTTTTTCGGTGAAG GAAAGGAGACTAACAGAGACGAATCTTTCTATGGTGATTTTGTTTT GGCTTATGATATATTATTGAAAGTTGACCACATTTACGATGCTATT AGAAACTACGTTACACAGAAGCCATACTCTAAGGACAAGTTCAAA TTGTACTTTCAAAACCCTCAGTTCATGGGTGGTTGGGATAAAGAC AAAGAAACAGATTATAGAGCTACTATTTTGAGATACGGTTCAAAAT ACTACTTAGCTATTATGGATAAGAAATATGCAAAATGCTTGCAGAA AATAGATAAAGACGATGTTAATGGTAATTATGAAAAAATTAATTATA AATTATTACCAGGACCAAACAAGATGTTACCAAAAGTTTTCTTTTC TAAAAAGTGGATGGCTTACTATAACCCTTCTGAAGACATACAGAAA ATTTACAAGAATGGTACATTTAAAAAGGGTGATATGTTTAATTTAAA TGACTGCCATAAGTTGATAGACTTCTTCAAAGATTCAATATCAAGG TATCCAAAATGGTCTAATGCATATGACTTTAATTTTTCAGAGACTG AGAAATATAAGGACATTGCTGGTTTCTATAGAGAAGTCGAAGAAC AAGGTTACAAGGTTTCTTTTGAGTCTGCTTCAAAGAAAGAAGTTGA TAAGTTGGTTGAAGAAGGTAAATTGTACATGTTTCAAATTTACAAC AAGGATTTTTCAGACAAATCTCATGGTACACCTAATTTGCACACAA TGTATTTCAAATTGTTGTTTGATGAAAACAACCACGGTCAGATAAG GTTGTCTGGTGGAGCTGAATTGTTTATGAGAAGAGCATCTTTGAA AAAGGAGGAGTTGGTCGTCCATCCTGCAAACTCTCCAATTGCTAA CAAGAACCCAGATAATCCAAAAAAGACTACTACATTGTCTTATGAC GTTTACAAGGATAAGAGATTCTCTGAGGATCAATATGAATTACATA TTCCAATTGCTATTAACAAGTGTCCAAAAAATATTTTCAAAATTAAT ACAGAGGTCAGAGTTTTATTAAAACACGATGATAACCCTTATGTCA TTGGTATAGATAGGGGTGAAAGGAACTTGTTATATATTGTTGTCGT CGATGGTAAAGGTAATATTGTCGAACAATACTCTTTAAATGAGATT ATAAACAACTTTAATGGAATTAGAATTAAGACAGATTATCATTCATT GTTAGATAAGAAAGAGAAGGAAAGATTTGAAGCTAGACAAAATTG GACATCTATTGAAAATATTAAAGAATTGAAGGCTGGATACATTTCA CAAGTTGTCCATAAAATATGTGAATTAGTTGAAAAATACGATGCTG TTATTGCTTTGGAAGATTTGAACTCTGGTTTTAAGAATTCTAGGGT TAAAGTTGAGAAACAGGTTTATCAAAAGTTTGAAAAAATGTTAATA GATAAGTTGAACTATATGGTTGACAAAAAATCTAACCCTTGCGCTA CAGGTGGAGCTTTGAAAGGTTATCAAATAACTAACAAGTTCGAAT CATTTAAGTCAATGTCTACTCAAAATGGTTTCATTTTCTATATTCCT GCTTGGTTAACATCAAAGATTGACCCATCAACTGGTTTTGTCAATT TGTTGAAAACTAAGTACACTTCTATAGCAGATTCAAAAAAGTTCAT TTCATCTTTCGATAGAATTATGTATGTTCCTGAGGAGGATTTATTT GAGTTTGCTTTGGATTACAAAAATTTTTCTAGAACAGATGCTGATT ACATAAAGAAGTGGAAATTGTACTCTTACGGTAATAGAATTAGGAT TTTCAGAAACCCTAAGAAAAATAATGTTTTCGACTGGGAAGAAGTT TGCTTGACATCTGCTTATAAAGAATTGTTTAATAAATATGGAATAAA CTATCAACAAGGTGATATTAGAGCTTTATTGTGTGAACAGTCTGAC AAGGCTTTTTATTCTTCATTTATGGCTTTGATGTCATTAATGTTGCA 


\begin{tabular}{|c|c|}
\hline & GATGAGAAATTCTATTACAGGTAGAACTGACGTCGATTTCTTGATA \\
\hline & TCTCCAGTTAAAAATTCTGATGGAATTTTCTATGATTCAAGAAACT \\
\hline & ATGAAGCTCAAGAGAATGCTATTTTACCTAAAAATGCTGATGCTAA \\
\hline & CGGAGCATATAATATTGCAAGGAAAGTCTTATGGGCTATTGGTCA \\
\hline & ATTTAAGAAAGCTGAAGACGAAAAATTAGACAAAGTTAAAATAGCT \\
\hline & ATTTCTAATAAAGAATGGTTAGAATACGCTCAAACTTCTGTTAAAC \\
\hline & $\mathrm{AC}$ \\
\hline linker_yo_VPR & TCTCCAGGTATCAGAAGATTGGACGCTTTGATCTCTACTTCTTTGT \\
\hline & ACAAGAAGGCTGGTTACAAGGAAGCTTCTGGTTCTGGTAGAGCT \\
\hline & GACGCTTTGGACGACTTCGACTTGGACATGTTGGGTTCTGACGCT \\
\hline & TTGGACGACTTCGACTTGGACATGTTGGGTTCTGACGCTTTGGAC \\
\hline & GACTTCGACTTGGACATGTTGGGTTCTGACGCTTTGGACGACTTC \\
\hline & GACTTGGACATGTTGATCAACTCTAGATCTTCTGGTTCTCCAAAGA \\
\hline & AGAAGAGAAAGGTTGGTTCTCAATACTTGCCAGACACTGACGACA \\
\hline & GACACAGAATCGAAGAAAAGAGAAAGAGAACTTACGAAACTTTCA \\
\hline & AGTCTATCATGAAGAAGTCTCCATTCTCTGGTCCAACTGACCCAA \\
\hline & GACCACCACCAAGAAGAATCGCTGTTCCATCTAGATCTTCTGCTT \\
\hline & СTGTTCCAAAGCCAGCTCCACAАCCATACCCATTCАСTTCTTCTTT \\
\hline & GTCTACTATCAACTACGACGAATTCCCAACTATGGTTTTCCCATCT \\
\hline & GGTCAAATCTCTCAAGCTTCTGCTTTGGCTCCAGCTCCACCACAA \\
\hline & GTTTTGCCACAAGCTCCAGCTCCAGCTCCAGCTCCAGCTATGGTT \\
\hline & TCTGCTTTGGCTCAAGCTCCAGCTCCAGTTCCAGTTTTGGCTCCA \\
\hline & GGTCCACCACAAGCTGTTGCTCCACCAGCTCCAAAGCCAACTCAA \\
\hline & GCTGGTGAAGGTACTTTGTCTGAAGCTTTGTTGCAATTGCAATTC \\
\hline & GACGACGAAGATTTGGGTGCTTTGTTGGGTAACTCTACTGACCCA \\
\hline & GCTGTTTTCACTGACTTGGCTTCTGTTGACAACTCTGAATTCCAAC \\
\hline & AATTGTTGAACCAAGGTATCCCAGTTGCTCCACACACTACTGAAC \\
\hline & CAATGTTGATGGAATACCCAGAAGCTATCACTAGATTGGTTACTG \\
\hline & GTGCTCAAAGACCACCAGACCCAGCTCCAGCTCCATTGGGTGCT \\
\hline & CCAGGTTTGCCAAACGGTTTGTTGTCTGGTGACGAAGATTTCTCT \\
\hline & TCTATCGCTGACATGGACTTCTCTGCTTTGTTGGGTTCTGGTTCTG \\
\hline & GTTCTAGAGACTCTAGAGAAGGTATGTTCTTGCCAAAGCCAGAAG \\
\hline & CTGGTTCTGCTATCTCTGACGTTTTCGAAGGTAGAGAAGTTTGTC \\
\hline & AACCAAAGAGAATCAGACCATTCCACCCACCAGGTTCTCCATGGG \\
\hline & CTAACAGACCATTGCCAGCTTCTTTGGCTCCAACTCCAACTGGTC \\
\hline & CAGTTCACGAACCAGTTGGTTCTTTGACTCCAGCTCCAGTTCCAC \\
\hline & AACCATTGGACCCAGCTCCAGCTGTTACTCCAGAAGCTTCTCACT \\
\hline & TGTTGGAAGATCCAGACGAAGAAACTTCTCAAGCTGTTAAGGCTT \\
\hline & TGAGAGAAATGGCTGACACTGTTATCCCACAAAAGGAAGAAGCTG \\
\hline & СTATCTGTGGTCAAATGGACTTGTCTCACCCACCACCAAGAGGTC \\
\hline & ACTTGGACGAATTGACTACTACTTTGGAATCTATGACTGAAGATTT \\
\hline & GAACTTGGACTCTCCATTGACTCCAGAATTGAACGAAATCTTGGA \\
\hline & CACTTTCTTGAACGACGAATGTTTGTTGCACGCTATGCACATCTCT \\
\hline
\end{tabular}


ACTGGTTTGTCTATCTTCGACACTTCTTTGTTC

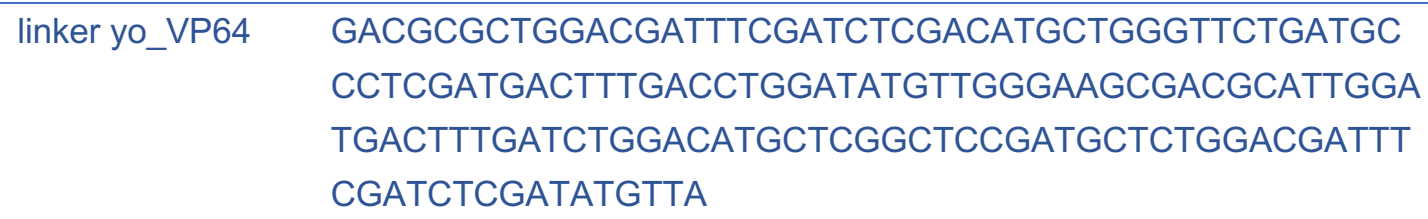

\begin{tabular}{ll}
\hline RGR-HDV & $\begin{array}{l}\text { Ggccggcatggtcccagcctcctcgctggcgccggctgggcaacatgcttcggcatggcgaat } \\
\text { gggac }\end{array}$ \\
\hline RGR-mHH & CTGATGAGTCCGTGAGGACGAAACGAGTAAGCTCGTC \\
\hline $\begin{array}{l}\text { crRNA spacer } \\
\text { binding lexOpR } \\
\text { anti-sense strand } \\
\text { (bA) }\end{array}$ & CATAACTGTATATACACCCA \\
\hline $\begin{array}{l}\text { crRNA spacer } \\
\text { binding lexOpR } \\
\text { sense strand (bS) }\end{array}$ & CCCTGGGTGTATATACAGTTA \\
\hline $\begin{array}{l}\text { Scrambled } \\
\text { crRNA for } \\
\text { denAsCas12a }\end{array}$ & TAATTTCTACTCTTGTAGATAATTGTGAGCGCTCACAATT \\
\hline $\begin{array}{l}\text { Scrambled } \\
\text { crRNA for } \\
\text { dLbCas12a }\end{array}$ & TAATTTCTACTAAGTGTAGATAAGTCTTCGTCTGAAGACAA \\
\hline $\begin{array}{l}\text { Scrambled } \\
\text { crRNA for } \\
\text { dMbCas12a }\end{array}$ & AAATTTCTACTGTTTGTAGATaagtcttcgtctgaagacaa \\
\hline $\begin{array}{l}\text { Pre-crRNA for } \\
\text { dLbCas12a }\end{array}$ & $\begin{array}{l}\text { TAATTTCTACTAAGTGTAGATCATAACTGTATATACACCCATAATTT } \\
\text { direct repeat }\end{array}$ \\
\hline
\end{tabular}




\begin{tabular}{|c|c|}
\hline $\begin{array}{l}\text { Repression/Gene } \\
\text { editing target site }\end{array}$ & CATCATGGCTGACAAACAAA \\
\hline T2A & $\begin{array}{l}\text { AGAGCAGAAGGAAGGGGTTCTTTGTTGACTTGTGGAGATGTTGA } \\
\text { GGAGAATCCAGGACCA }\end{array}$ \\
\hline P2A & $\begin{array}{l}\text { GGTTCTGGTGCTACTAACTTCTCTTTGTTGAAGCAAGCTGGTGAC } \\
\text { GTTGAAGAAAACCCAGGTCCA }\end{array}$ \\
\hline yo_AcrVA1 & $\begin{array}{l}\text { TATGAAGCTAAAGAAAGATATGCTAAAAAAAAAATGCAAGAAAACA } \\
\text { CCAAGATCGACACCCTTACCGACGAACAGCATGATGCCTTGGCT } \\
\text { CAGTTGTGCGCCTTTAGACACAAGTTCCACTCTAACAAGGATAGT } \\
\text { TTGTTTTTGTCTGAATCTGCTTTCTCTGGTGAATTTTCTTTTGAAAT } \\
\text { GCAATCTGACGAGAACAGTAAGTTGAGGGAGGTCGGTTTGCCAA } \\
\text { CCATCGAGTGGAGTTTCTACGACAACTCTCATATTCCAGATGATTC } \\
\text { TTTTAGAGAATGGTTTAATTTCGCTAACTACTCTGAGCTTTCTGAA } \\
\text { ACCATCCAAGAACAAGGTCTTGAATTGGACTTGGACGATGACGAA } \\
\text { ACCTACGAGCTTGTCTACGACGAATTGTACACCGAGGCCATGGG } \\
\text { TGAATACGAGGAGTTGAACCAAGATATTGAGAAGTATTTGAGGAG } \\
\text { GATCGACGAGGAGCATGGTACCCAGTACTGCCCAACCGGTTTTG } \\
\text { CCAGATTGAGA }\end{array}$ \\
\hline yo_AcrVA4 & $\begin{array}{l}\text { TATGAAATTAAGTTGAACGACACCTTGATCCACCAAACCGACGAC } \\
\text { AGAGTCAACGCTTTCGTCGCCTATAGATACTTGCTTAGGAGGGGT } \\
\text { GACCTTCCTAAGTGCGAGAACATCGCTAGAATGTACTACGACGGA } \\
\text { AAAGTCATCAAGACCGACGTCATCGACCATGACAGTGTTCACTCT } \\
\text { GACGAGCAAGCTAAGGTCTCTAACAACGATATCATCAAAATGGCT } \\
\text { ATTAGTGAATTGGGTGTTAATAATTTTAAATCTTTGATCAAGAAACA } \\
\text { AGGTTACCCATTCTCTAACGGTCACATCAACTCTTGGTTCACCGA } \\
\text { CGATCCAGTCAAGAGTAAGACCATGCATAATGACGAGATGTACTT } \\
\text { GGTTGTCCAAGCTTTGATTAGAGCTTGTATCATCAAGGAAATCGAT } \\
\text { TTGTATACTGAACAATTGTATAATATTATTAAATCTTTGCCTTACGA } \\
\text { CAAGAGGCCAAACGTCGTCTACTCTGACCAGCCTTTGGACCCAAA } \\
\text { CAACTTGGACCTTTCTGAGCCAGAGCTTTGGGCTGAACAAGTTGG } \\
\text { TGAATGCATGAGATACGCTCACAACGACCAGCCTTGCTTCTACAT } \\
\text { CGGATCTACCAAGAGGGAGCTTAGGGTCAACTACATCGTCCCAG } \\
\text { TCATCGGTGTCAGAGACGAGATCGAAAGGGTCATGACCTTGGAA } \\
\text { GAAGTTAGAAATTTGCACAAG }\end{array}$ \\
\hline yo_AcrVA5 & $\begin{array}{l}\text { AAGATCGAATTGTCTGGTGGTTACATCTGTTACTCTATCGAAGAAG } \\
\text { ACGAAGTTACTATCGACATGGTTGAAGTTACTACTAAGAGACAAG } \\
\text { GTATCGGTTCTCAATTGATCGACATGGTTAAGGACGTTGCTAGAG } \\
\text { AAGTTGGTTTGCCAATCGGTTTGTACGCTTACCCACAAGACGACT }\end{array}$ \\
\hline
\end{tabular}


CTATCTCTCAAGAAGACTTGATCGAATTCTACTTCTCTAACGACTT CGAATACGACCCAGACGACGTTGACGGTAGATTGATGAGATGGT CT

\begin{tabular}{|c|c|}
\hline Tsynth24 & $\begin{array}{l}\text { TGGGTGGTATGTTATATAACTGTCTAGAAATAAAGAGTATCATCTT } \\
\text { TCAAA }\end{array}$ \\
\hline SUP4t & TTTTTTTGTTTTTTATGTCT \\
\hline ADH1t & $\begin{array}{l}\text { GCGAATTTCTTATGATTTATGATTTTTATTATTAAATAAGTTATAAAA } \\
\text { AAAATAAGTGTATACAAATTTTAAAGTGACTCTTAGGTTTTAAAAC } \\
\text { GAAAATTCTTATTCTTGAGTAACTCTTTCCTGTAGGTCAGGTTGCT } \\
\text { TTCTCAGGTATAGCATGAGGTCGCTCTTATTGACCACACCTCTAC } \\
\text { CGGCATG }\end{array}$ \\
\hline Tysnth6 & TATATATTTAATAAAGAGTATCATCTTTCAAA \\
\hline CYC1t & $\begin{array}{l}\text { CATGTAATTAGTTATGTCACGCTTACATTCACGCCCTCCCCCCACA } \\
\text { TCCGCTCTAACCGAAAAGGAAGGAGTTAGACAACCTGAAGTCTAG } \\
\text { GTCCCTATTTATTTTTTTATAGTTATGTTAGTATTAAGAACGTTATTT } \\
\text { ATATTTCAAATTTTTCTTTTTTTTCTGTACAGACGCGTGTACGCATG } \\
\text { TAACATTATACTGAAAACCTTGCTTGAGAAGGTTTTGGGACGCTC } \\
\text { GAAGGCTTTAATTTGCAAGCT }\end{array}$ \\
\hline mTGUO1 & TATATAACTGTCTAAAATAAAGAGTATCATCTAAAAA \\
\hline
\end{tabular}

